\title{
Oral Administration of Quercetin or Its Derivatives Inhibit Bone Loss in Animal Model of Osteoporosis
}

\author{
Yue-Yue Huang, ${ }^{1}$ Zi-Hao Wang, ${ }^{2}$ Li-Hui Deng, ${ }^{2}$ Hong Wang $\mathbb{D}^{2}$, and Qun Zheng $\mathbb{D}^{2}$ \\ ${ }^{1}$ Department of Hematology, The Second Affiliated Hospital and Yuying Children's Hospital of Wenzhou Medical University, \\ Wenzhou, China \\ ${ }^{2}$ Department of Rheumatology Immunology, The Second Affiliated Hospital and Yuying Children's Hospital of Wenzhou \\ Medical University, Wenzhou, China \\ Correspondence should be addressed to Hong Wang; 202020@wzhealth.com and Qun Zheng; 344457512@qq.com
}

Received 14 March 2020; Accepted 31 March 2020; Published 28 October 2020

Guest Editor: Marcos R. de Oliveira

Copyright ( 92020 Yue-Yue Huang et al. This is an open access article distributed under the Creative Commons Attribution License, which permits unrestricted use, distribution, and reproduction in any medium, provided the original work is properly cited.

\begin{abstract}
Objectives. Quercetin (Q) and its derivatives are the major members of the naturally occurring flavonoid family, which possess beneficial effects on disease prevention including osteoporosis. The present study is aimed at further investigating the efficacy of the $\mathrm{Q}$ and its derivatives on bone pathology, bone-related parameters under imageology, bone maximum load, and serum bone metabolism indexes in animal model of osteoporosis. Potential mechanisms of $\mathrm{Q}$ and its derivatives in the treatment of osteoporosis as well as the existing problems regarding the modeling method and limitations of researches in this area were also summarized. Eight databases were searched from their inception dates to February 2020. Nineteen eligible studies containing 21 comparisons were identified ultimately. The risk of bias and data on outcome measures were analyzed by the CAMARADES $10-$ item checklist and Rev-Man 5.3 software separately. The results displayed the number of criteria met varied from 3/10 to 7/10 with an average of 5.05. The present study provided the preliminary preclinical evidence that oral administration of Q or its derivatives was capable of improving bone pathology, bone-related parameters under imageology and bone maximum load, increasing serum osteocalcin, alkaline phosphatase, and estradiol, and reducing serum c-terminal cross-linked telopeptide of type I collagen $(P<0.05)$. No statistical difference was seen in survival rate, index of liver, or kidney function $(P>0.05)$. Q and its derivatives partially reverse osteopenia probably via antioxidant, anti-inflammatory, promoting osteogenesis, inhibiting osteoclasts, and its estrogen-like effect. The findings reveal the possibility of developing Q or its derivatives as a drug or an ingredient in diet for clinical treatment of osteoporosis.
\end{abstract}

\section{Introduction}

Osteoporosis, as a systemic skeletal disease characterized by loss of bone mass and bone microarchitectural deterioration, causes enhanced bone fragility and a greater risk of fractures [1]. Some risk factors are bound up with osteopathic fracture, containing age, race, cigarette smoking, low physical activity, hormone-related factors, the use of drugs (e.g., glucocorticoids), low calcium and vitamin D levels, and prior history of fracture [2]. It gradually becomes a major public health issue affecting exceeded 200 million people worldwide [3], and the burden of society is continuously increasing with the aging of the world population [4]. Thus, how to effec- tively prevent and treat osteoporosis has attracted more and more attention of the world.

Physical activity [5], supplementing with calcium and vitamin $\mathrm{D},[6,7]$ and pharmacological therapies [8] (including estrogen [9], bisphosphonates [10], denosumab [11], or teriparatide [12]) are the most commonly used clinical approaches for the prevention or treatment of osteoporosis. However, there is no definite solution for this disease because there are still some problems in the above treatment methods [13]. For example, although bisphosphonates are widely used globally as the main treatment for osteoporosis, it does not include proven efficacy against nonvertebral fractures, and the evidence for benefit beyond 5 years in intervention 
studies is limited [14]. Besides, there are concerns that atypical femur fractures and osteonecrosis of the jaw may be caused by long-term use of bisphosphonates [14]. Highimpact exercise (e.g., running) is conducive to producing changes that reflect distal tibial bone adaptation [5]. However, a well-designed training intervention is difficult to achieve in normal people much less in patients with osteoporosis or fracture. The benefits of supplementing with vitamin $\mathrm{D}$ and calcium for fracture prevention and holistic fall remain uncertain [15]. There are inconsistent findings for adverse effects on the cardiovascular system of calcium supplements with or without vitamin D [7, 16, 17], and the adverse effects on the skeleton and musculature of highdose vitamin $\mathrm{D}$ were reported by recent evidence $[18,19]$. Despite its excellent antiosteoporosis effect for postmenopausal osteoporosis, long-term use of estrogen for osteoporosis caused many side effects, containing an increased incidence of cardiovascular accidents, endometrial cancer [20-22], as well as the loss of mesenteric ganglion neurons and dominant ovarian follicles [23]. Therefore, obtaining a new effective drug without side effects is needed urgently in the treatment of osteoporosis.

Quercetin (Q, $\mathrm{C}_{15} \mathrm{H}_{10} \mathrm{O}_{7}$, Figure $1(\mathrm{a})$ ) and its derivatives (Figures 1(b) and 1(c)) are the major members of the naturally occurring flavonoid family. Flavonoid is well known for its antioxidant and anti-inflammatory properties. Q and its derivatives have been widely used as a natural antioxidant in the treatment of cardiovascular diseases, tumor, and some metabolic diseases including osteoporosis for many years in China $[24,25]$. Recent studies $[26,27]$ demonstrate that $\mathrm{Q}$ and its derivatives are potent natural osteogenic agents by multiple pharmacological activities including the function of antioxidant, anti-inflammatory, and estrogen-like effect in vivo and vitro studies. However, the scattered evidence and uncertain mechanisms limited the application of $\mathrm{Q}$ and its derivatives in the clinic. Systematic review and metaanalysis of animal studies are considered to be a valuable tool to provide important insights into the validity of animal studies, improve the precision of estimated effects, and support further generalization to human clinical trials [28]. Thus, the present study is aimed at investigating the preclinical evidence and possible mechanisms of $\mathrm{Q}$ and its derivatives in animal models of osteoporosis.

\section{Methods}

2.1. Data Sources and Search Strategies. A comprehensive literature search about animal experimental studies of Q or its derivatives for osteoporosis was conducted in the Chinese Science and Technology Journal Database, WanFang, China National Knowledge Infrastructure, Chinese Biomedical Database, PubMed, EMBASE, Cochrane library, and Web of Science database from their inception dates to February 2020. The following search terms were used in PubMed and were modified to suit other databases: "Quercetin" AND "Osteoporosis OR Bone Loss". In addition, reference lists from the resulting publications and reviews were also searched carefully for the eligible studies.
2.2. Eligibility Criteria and Data Extraction. Two authors (Yue-Yue Huang and Zi-Hao Wang) selected the studies separately by browsing the abstracts and full texts via the eligibility criteria. The study was included if it met the following criteria: (1) controlled studies assessing the administration of $\mathrm{Q}$ and its derivatives for osteoporosis or bone loss animal models established by various ways; (2) the treatment group received $\mathrm{Q}$ or its derivatives as monotherapy with unrestricted dosage, medicament type, route of administration, and time for the medicine application. Blank treatment or isasteric placebo was received in the control group; (3) the present study received bone pathology and/or bone mineral density (including femur bone mineral density (F-BMD), lumbar spine bone mineral density (L-BMD)) and/or bone histomorphometric parameters under micro-CT (trabecular number (Tb.N), trabecular thickness (Tb.Th)) and/or bone maximum load and/or bone turnover markers (serum alkaline phosphatase (ALP), C-terminal cross-linked telopeptide of type I collagen (CTX) and osteocalcin (OC)) and/or serum estradiol and/or uterine weight and/or indicators of adverse reactions as the primary outcome measures, while the antiosteoporosis mechanisms of Q or its derivatives was selected as the second outcome measures. Exclusion criteria was as below: (1) other types of studies (in vitro studies, case reports, clinical trials, reviews, abstracts, comments, and editorials); (2) combination with other compounds; (3) compared with other traditional Chinese medicine; (4) no any primary outcome indicator were involved or incomplete date; (5) inconsistent of graphic and textual data; (6) no control group; (7) duplicate publications; (8) not osteoporosis or bone loss model.

The details were extracted from included studies by two independent authors (Yue-Yue Huang and Zi-Hao Wang) using a predefined form. The information included the authors and years of publication; information of animals; modeling method; the use of anesthetics anaesthetize in the course of the experiment; the therapeutic regimen of treatment and control group; and primary and/or secondary outcomes and its intergroup differences. Only data from the osteoporosis group and Q+osteoporosis groups were included for analysis when a study is involved in multiple intervention groups. When the outcomes were displayed through gradient doses of drug therapy or determined at different times, only the data of the highest dose and the final measurement was included for analysis.

2.3. Risk of Bias in Individual Studies. CAMARADES 10-item quality checklist [29] with minor modification was used to assess the study quality by two independent authors (Yue-Yue Huang and Li-Hui Deng). The modification is listed as follows: D: blinded induction of model (group randomly after modeling); F: use of anesthetic without significant bony protective activity or nephrotoxicity; G: appropriate animal model with complications or risk factors (including age, hyperlipemia, diabetes, or hypertensive). Disagreements in the process of selecting studies, extracting data, and assessing the quality of studies were resolved by consensus or arbitration by the correspondence author (Qun Zheng). 


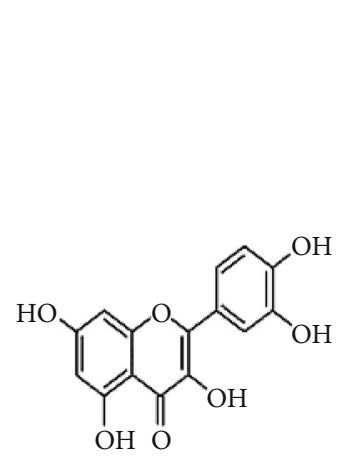

(a)

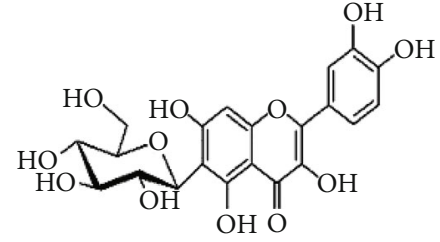

(b)

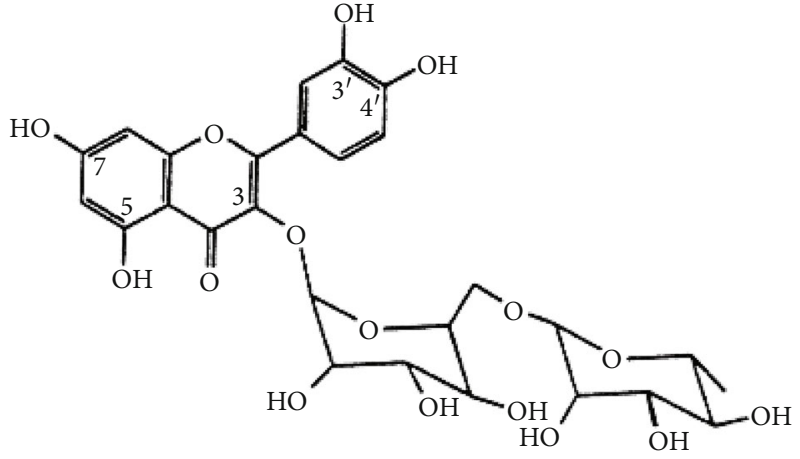

(c)

FIgUre 1: The chemical structure of $\mathrm{Q}$ and its derivatives.

2.4. Statistical Analysis. The RevMan 5.3 software was used for data analysis where possible; otherwise, comparison between groups was performed. The bar graphs were drawn via Prism 6. In meta-analysis, standardized mean differences (SMDs) and 95\% confidence intervals (95\% CIs) were calculated to estimate the combined overall effect sizes when the outcomes were determined in various ways or the unit of measurement is different. Heterogeneity was assessed using the Cochrane Q-statistic test $(P<0.05$ was considered statistically significant) and the $I^{2}$-statistic test $\left(I^{2}<50 \%\right.$ was considered homogeneous). Random $\left(I^{2}>50 \%\right)$ or fixed-effects model $\left(I^{2}<50 \%\right)$ was selected according to the results of $I^{2}$. In order to ensure the reliability of results, a sensitivity analysis was performed, and potential publication bias was assessed by the visual inspection of the funnel plot and asymmetry test. Moreover, in order to explore the impact of potential confounding factors on the estimates of combined effect size, subgroup analyses were conducted in this study. The significance level was set at $P<0.05$.

\section{Results}

3.1. Study Selection. A total of 126 studies were identified by the initial database search and 19 eligible studies [4, 27, 3046] containing 21 comparisons were included in this study ultimately. The process followed for study selection is shown in Figure 2.

3.2. Characteristics of Included Studies. The detailed characteristics of the included studies were generalized in Table 1. Nine English studies and 8 Chinese studies between 2000 and 2019 with 414 animals were identified. The sample size of each study ranged from 14 to 40 animals. Female Sprague-Dawley (SD) rats $(51.5 \%)$, female Wistar rats (9.42\%), female Y59 rats $(4.7 \%)$, female C57BL/6 mice $(15.6 \%)$, male SD rats $(4.7 \%)$, male Wistar rats $(4.7 \%)$, and male C57BL/6 mice (9.42\%) were used in the studies. The weight of SD, Wistar, and Y59 rats varied between $170 \mathrm{~g}$ and $350 \mathrm{~g}$, and the weight of mice varied between $16.5 \mathrm{~g}$ and 18.8 g. Fourteen $[4,27,30,32-35,37,38,43-46]$ studies established osteoporosis or bone loss model by bilateral oophorectomy; 2 studies [39, 40] by feeding with high-fat diet for several weeks, 1 study [42] by intraperitoneal injec- tion of STZ (100 mg/kg), 1 study [31] by oral gavage of isotretinoin $(80 \mathrm{mg} / \mathrm{kg}, \mathrm{qd})$ for 14 days, 1 study [41] by subcutaneous injection of methylprednisolone sodium succinate ( $40 \mathrm{mg} / \mathrm{kg}$ body mass) for 6 weeks, and 1 study [36] by oral gavage of $\mathrm{n}-\mathrm{ZnO}(600 \mathrm{mg} / \mathrm{kg}$, qd) for 5 consecutive days. Anesthetics were reported in 13 studies. Of which, chloral hydrate was reported in 4 studies $[4,35,37,46]$, mixture of ketamine and xylazine in 2 studies [31, 41], ketamine in 2 studies [34, 44], sodium pentobarbital in 2 studies $[30,38]$, ether in 1 study [36], ethyl ether in 1 study [39], and diethyl ether in 1 study [42]. Detailed information of Q or its derivatives in each study is displayed in Table 2. All studies implemented different doses of $\mathrm{Q}$ or its derivatives by oral or intragastric administration. Among them, 14 studies [27, $30-38,41,42,44,45]$ reported the dose gradient of $Q$ ranged $5 \mathrm{mg} / \mathrm{kg} / \mathrm{d}$ to $300 \mathrm{mg} / \mathrm{kg} / \mathrm{d}, 2$ studies $[39,40]$ utilized a standard high-fat diet plus $0.01 \%$ Q per day, 1 study [43] utilized a standard high-fat diet plus $2.5 \%$ Q ( 5 g per mouse) per day, 1 study [27] utilized quercetin-6-C-A-D-glucopyranoside (QCG) with $5 \mathrm{mg} / \mathrm{kg} / \mathrm{d}$, and 2 studies $[4,46]$ utilized quercetin-3-O-rutinose with $2.5 \mathrm{mg} / \mathrm{kg} / \mathrm{d}$. Bone pathology was utilized as primary outcome measure in 3 studies [31, 38, 41], F-BMD in 13 studies [4, 27, 30-34, 39, 42-46], L-BMD in 3 studies [35, 43, 44], Tb.Th in 4 studies [27, 30, 33, 43], Tb.N in 4 studies $[27,30,33,43]$, bone maximum load in 9 studies $[27,32,34,37,39-42,45]$, serum ALP in 8 studies $[30,31,33-36,42,45]$, serum OC in 4 studies [35, 37, 41, 42], CTX in 3 studies [34, 35, 41], survival rate in 4 studies $[4,33,38,45]$, blood urea nitrogen (BUN) in 2 studies [31, 34 ], serum creatinine (SCr) in 1 study [34], aspartate aminotransferase (AST) in 1 study [31], and alanine aminotransferase (ALT) in 1 study [31]. Serum Superoxide dismutase (SOD) was reported as second outcome measure in 3 studies $[31,39,42]$; serum catalase (CAT) in 2 studies [31, 42]; serum malondialdehyde (MDA) in 3 studies [31, 39, 40]; serum glutathione peroxidase (GSH) in 4 studies [ $31,39,40,42]$; serum estradiol in 3 studies $[35,37,45]$; uterine weight in 3 studies $[27,33,43]$; serum tumor necrosis factor- $\alpha$ (TNF- $\alpha)$ in 3 studies [4, 32, 36]; interleukin-6 in 2 studies [4, 36]; interferon $\gamma$ (INF- $\gamma$ ) in 1 study [4]; C-reactive protein (CRP) in 1 study [36]; nuclear factor $-\kappa \mathrm{B}(\mathrm{NF}-\kappa \mathrm{B})$ in 1 study [32]; serum NO in 1 study [36]; serum $\mathrm{Ca}$ and $\mathrm{P}$ in 4 studies [30, $35,41,46]$; urinary $\mathrm{Ca}$ and $\mathrm{P}$ in 3 studies $[30,33,35,46]$; 


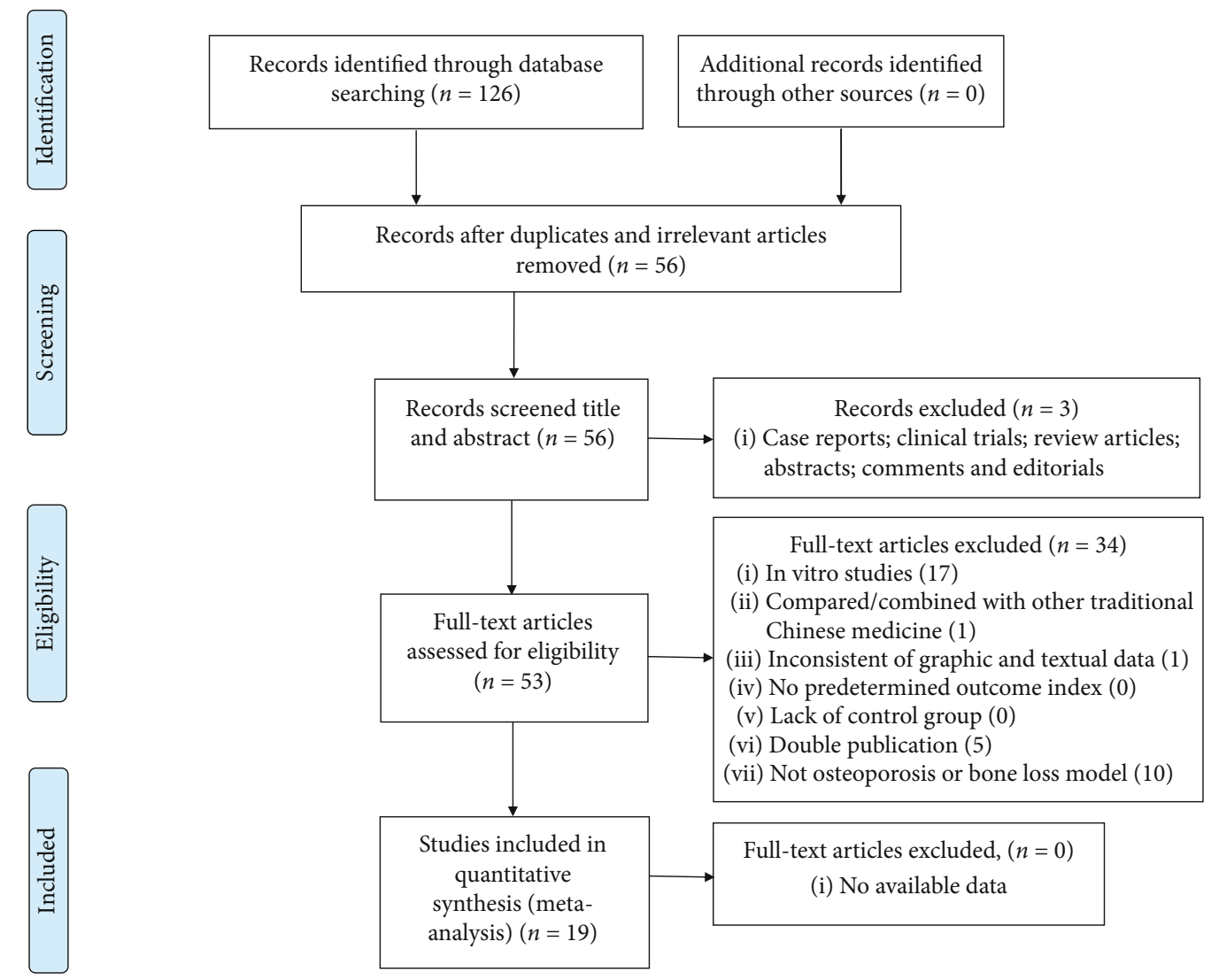

FIGURE 2: Summary of the process for identifying candidate studies.

haematological parameters in 1 study [31]; extracellular regulated protein kinases (ERK), amino-terminal protein kinas (JNK), and P38 in 1 study [34]; bone morphogenetic protein 2 (BMP2); and smad family member 4 (Smad4) in 1 study [35]; serum colla1, bone Gla protein 2 (Bglap2), NF-E2related factor 2 (Nrf-2), thyroid hormone receptor $\alpha 1$ (TR $\alpha 1$ ), and glycogen synthase kinase $3 \beta$ (GSK-3) in 1 study [39]; cathepsin K (CTSK) in 2 studies [39, 43]; receptor activator of nuclear factor- $\kappa \mathrm{B}$ (RANK) in 2 studies [27, 39]; Runt-related transcription factor 2 (Runx2) in 1 study [30]; Forkhead box transcription factor $\mathrm{O} 1$ (FoxO1) in 1 study [30]; macrophage colony-stimulating factor (M-CSF) and cfos in 1 study [27]; calcitonin receptor (CTR), matrix metalloproteinase 9 (MMP9); and nuclear factor of activated $\mathrm{T}$ cells c1 (NFATc1) in 1 study [43].

3.3. Study Quality. Detailed results of methodological quality are presented in Table 3. The number of criteria met in each study varied from $3 / 10$ to $7 / 10$ with the average of 5.05. Only 2 studies [30,39] were not a peer-reviewed publication, and 3 studies $[32,44,45]$ did not mention control of temperature. Two $[31,46]$ of the included studies did not declare randomization. The ways of blinding induction of model were reported in 5 studies $[4,31,35,36,43]$, and all of them reported the animals were grouped randomly after modeling. No study mentioned the calculation of sample size and none used a blinding method during outcome assessment and appropriate animal model. Thirteen studies $[4,30,31,34-39,41,42,44,46]$ used the anesthetic without protective and toxic effects on bones. Compliance with animal welfare regulations was not described in 3 studies [27, 38, 42,], and the potential conflict of interests was not mentioned in 7 studies [4, 30, 34, 39, 44-46].

\subsection{Effectiveness}

3.4.1. Bone Pathology. Three studies [31, 38, 41] utilized bone pathology as a primary outcome measure. Among them, 1 study [31] reported that osteoporotic rats treated with $Q$ showed marked the improvement of the structure of femoral cortical bone compared with osteoporotic rats induced by $13 \mathrm{cRA}$, which showed thickness was nearly similar to that of the control group although a few small intracortical cavities were still present. One study [38] reported that Q treatment was observed to prevent trabecular fracture and osteoblast apoptosis and maintain normal distribution of trabecular. Another study [41] reported that the administration of $150 \mathrm{mg} / \mathrm{kg} \mathrm{Q}$ increased femoral trabecular and cortical thickness by $36 \%$ and $22 \%$, respectively, compared with the osteoporotic rats induced by methylprednisolone sodium succinate.

3.4.2. Bone Related Parameters under Imageology and Bone Maximum Load. With dual-energy X-ray absorptiometry, meta-analysis of 15 researches [27, 31-34, 39, 42-46] and 3 researches $[4,30,35,43,44]$ separately showed a significant effect of Q or its derivatives for increasing F-BMD $(n=301$, 


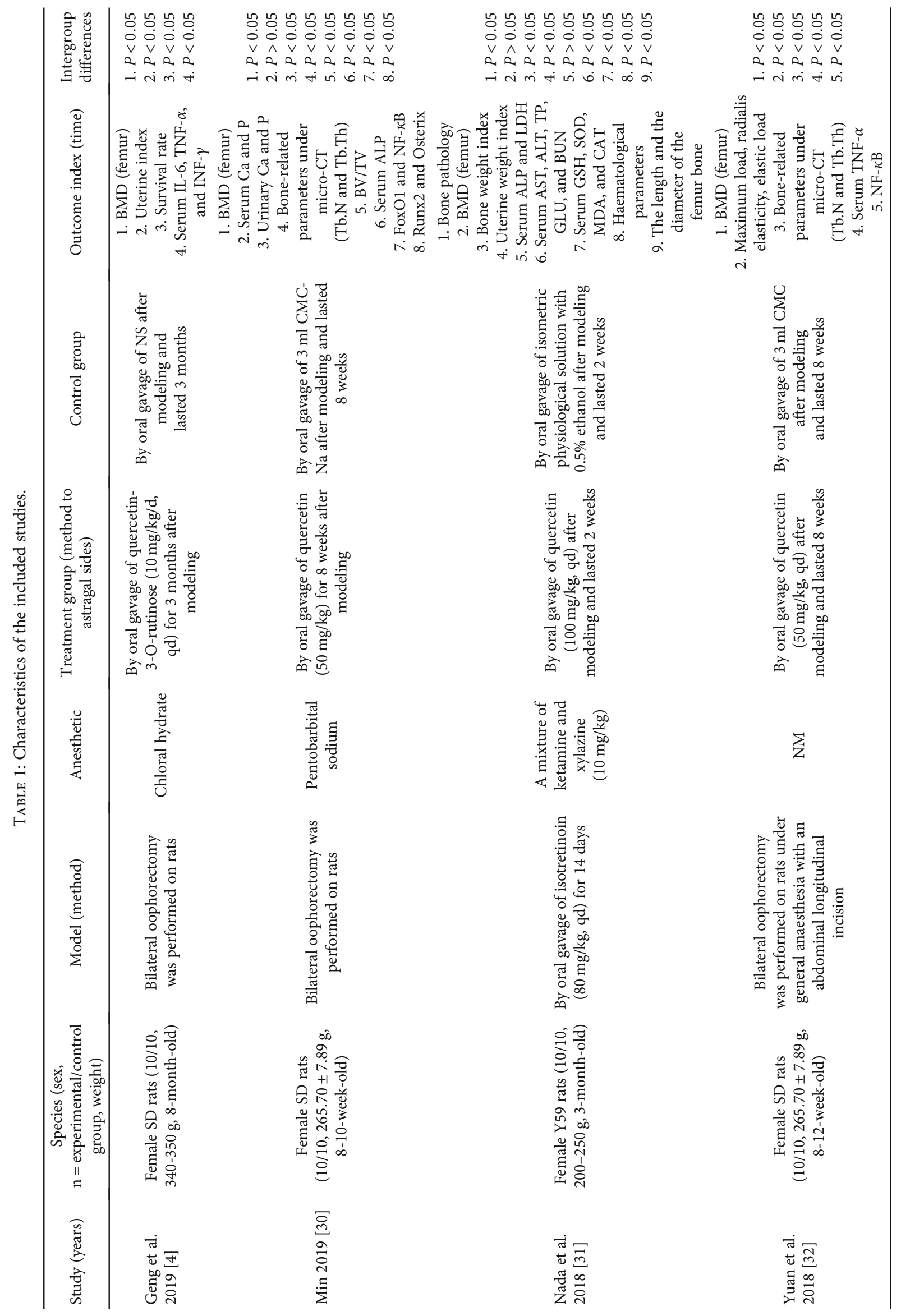




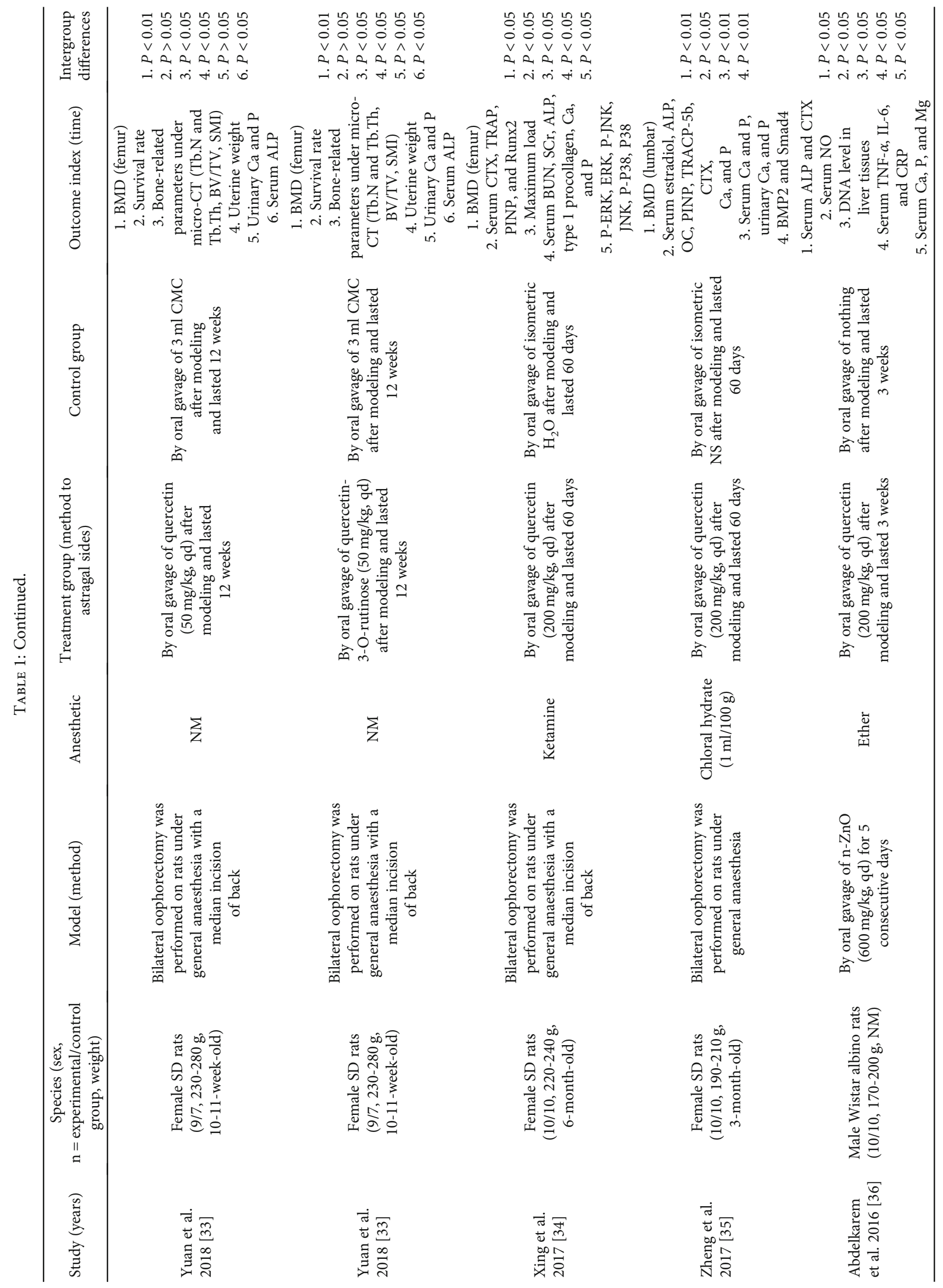




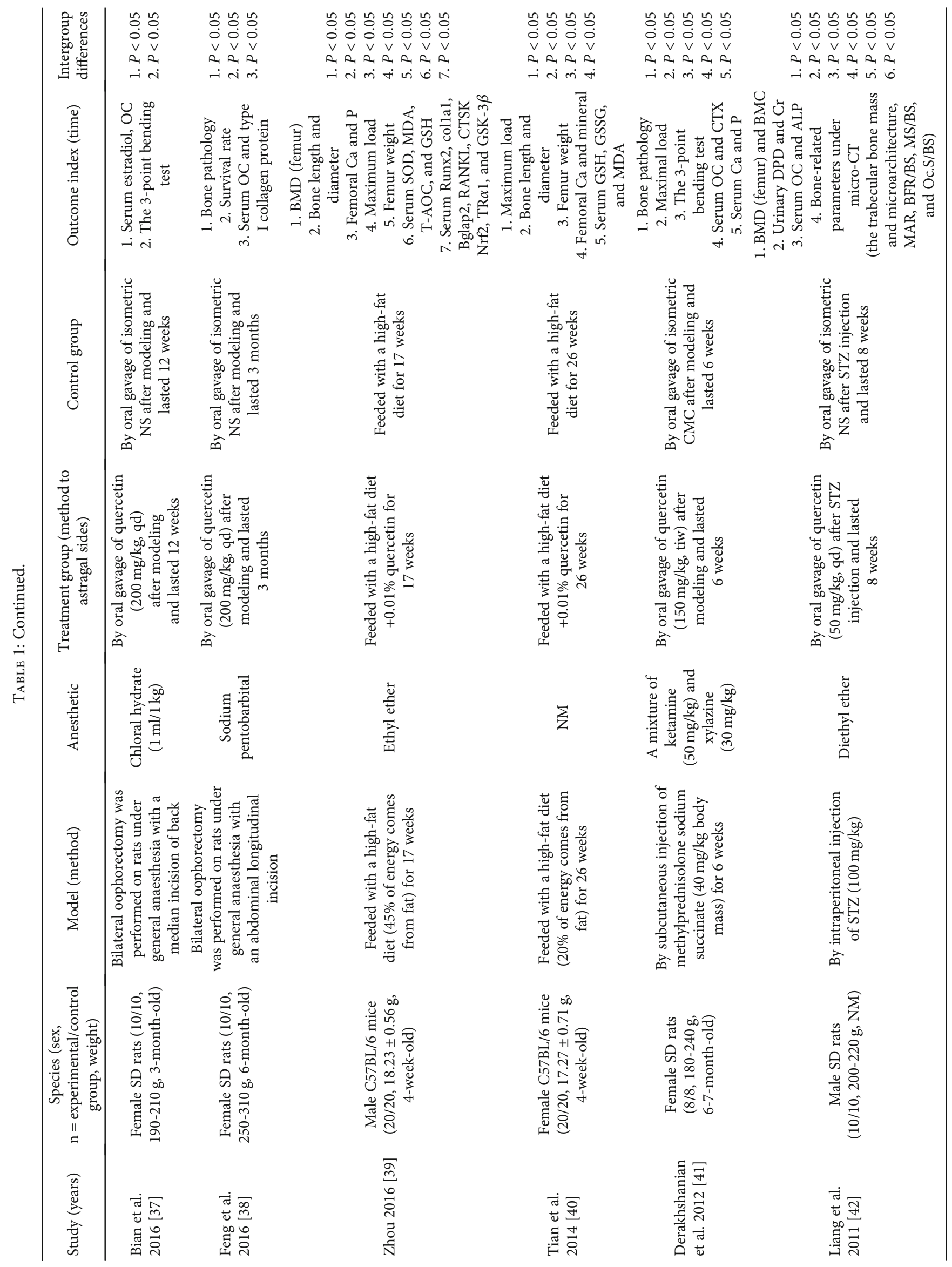




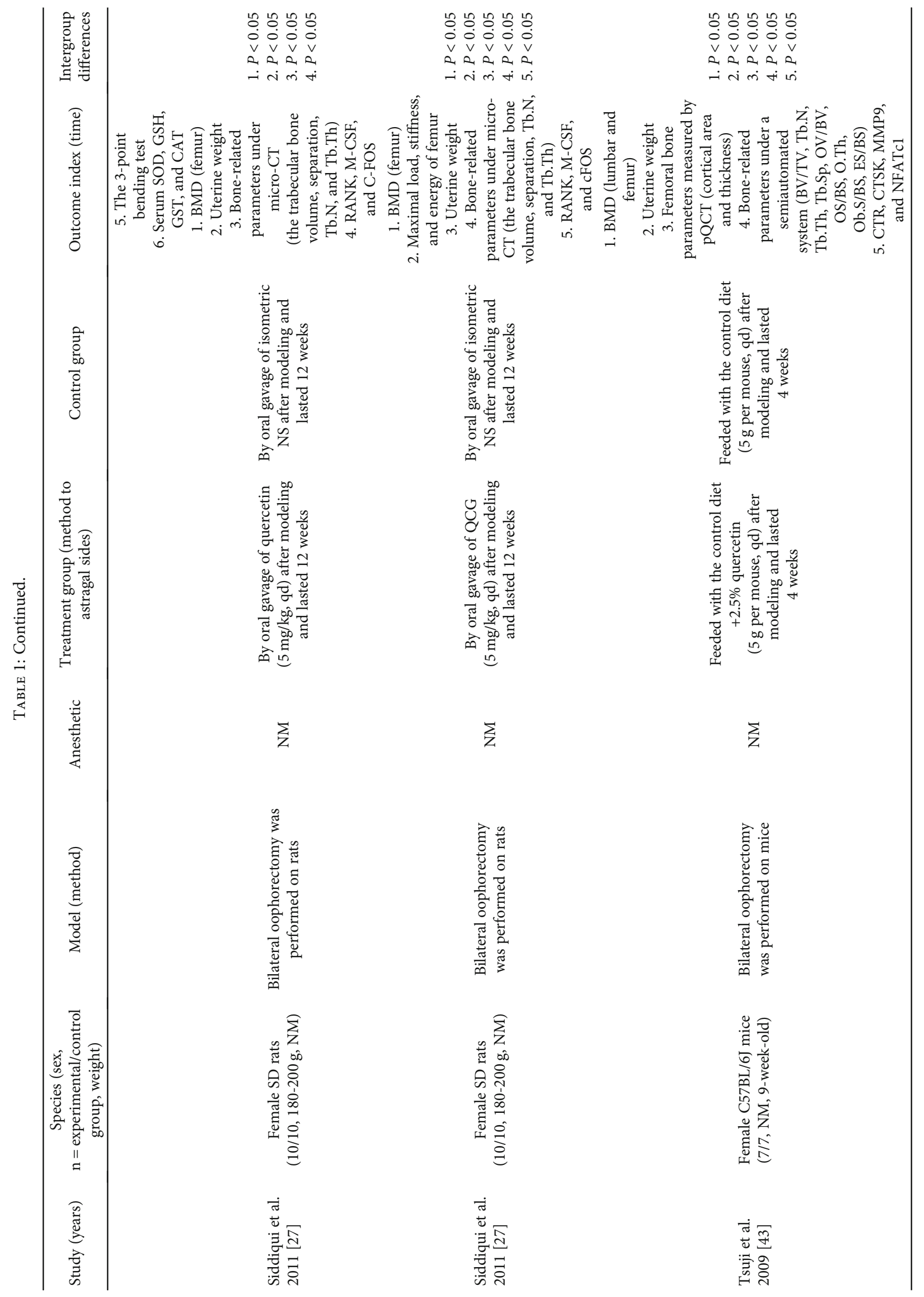




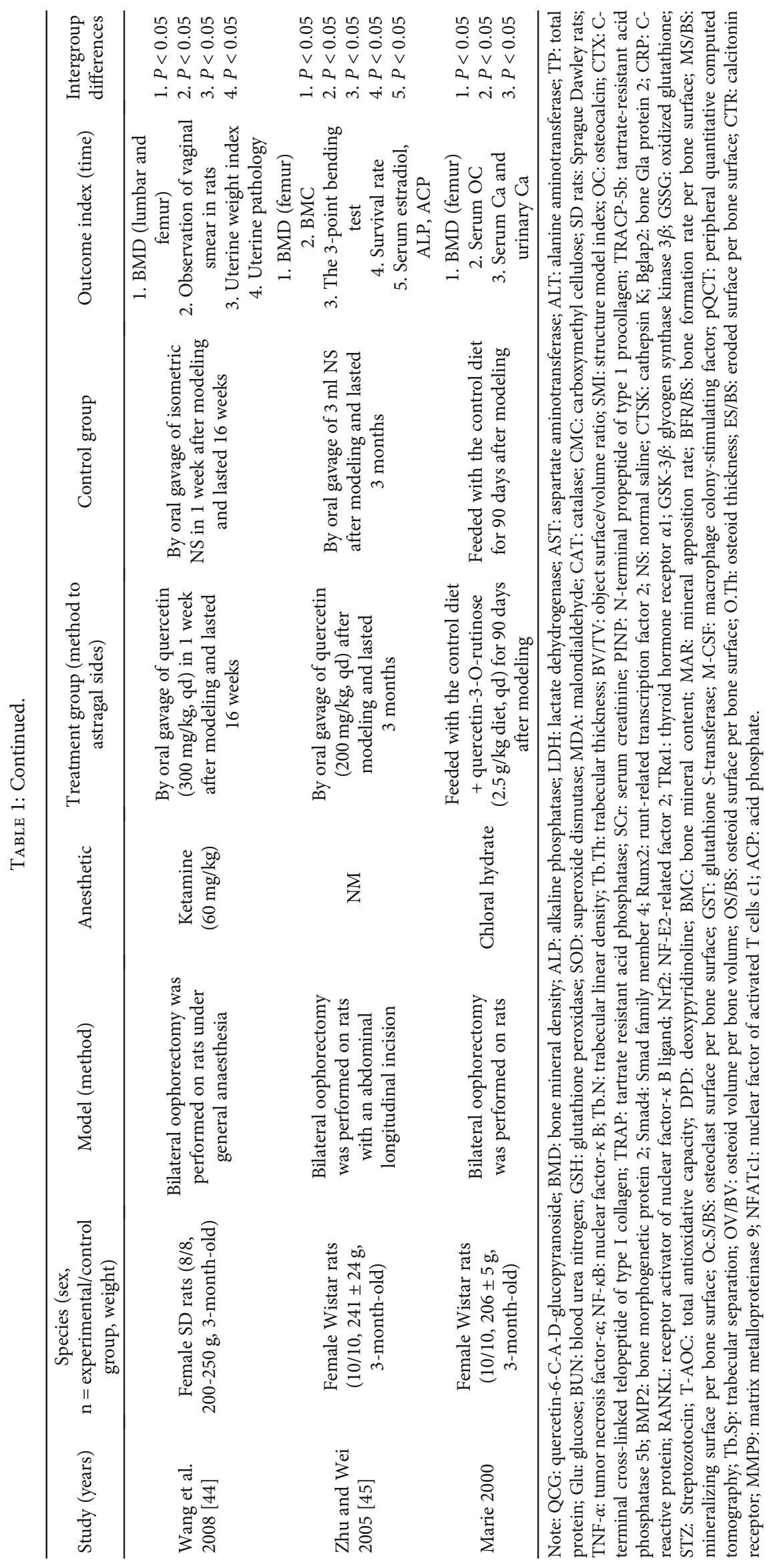


TABLE 2: Information on quercetin or its derivatives of each study.

\begin{tabular}{|c|c|c|c|c|}
\hline Study (years) & $\begin{array}{l}\text { Chemical } \\
\text { composition }\end{array}$ & Source & $\begin{array}{l}\text { Purity } \\
(\%)\end{array}$ & $\begin{array}{l}\text { Quality control } \\
\text { reported }\end{array}$ \\
\hline $\begin{array}{l}\text { Min et al. } \\
2019[30]\end{array}$ & Quercetin & Sigma-Aldrich Corporation, USA & $(\geq 99 \%)$ & $\begin{array}{l}\text { Batch number: } \\
\text { XSD201510008, } \\
\text { HPLC }\end{array}$ \\
\hline $\begin{array}{l}\text { Geng et al. } \\
2019[4]\end{array}$ & $\begin{array}{l}\text { Quercetin-3-O- } \\
\text { rutinose }\end{array}$ & $\begin{array}{l}\text { National Institute of controlled drugs and } \\
\text { biological products, China }\end{array}$ & $(\geq 98 \%)$ & HPLC \\
\hline $\begin{array}{l}\text { Nada et al. } \\
2018[31]\end{array}$ & Quercetin & Aldrich Ch. Co. Inc. Milwaukee WI, USA & $(98 \%)$ & $?$ \\
\hline $\begin{array}{l}\text { Yuan et al. } \\
2018[32]\end{array}$ & Quercetin & Sigma-Aldrich Corporation, USA & $(\geq 99 \%)$ & HPLC \\
\hline $\begin{array}{l}\text { Yuan et al. } \\
2018[33]\end{array}$ & Quercetin & Sigma-Aldrich Corporation, USA & $(\geq 99 \%)$ & $\begin{array}{l}\text { Batch number: } \\
\text { XSD201510008, } \\
\text { HPLC }\end{array}$ \\
\hline $\begin{array}{l}\text { Yuan et al. } \\
2018[33]\end{array}$ & $\begin{array}{l}\text { Quercetin-3-O- } \\
\text { rutinose }\end{array}$ & Sigma-Aldrich Corporation, USA & $(\geq 99 \%)$ & $\begin{array}{c}\text { Batch number: } \\
\text { XSD201510008, } \\
\text { HPLC }\end{array}$ \\
\hline $\begin{array}{l}\text { Xing et al. } \\
2017[34]\end{array}$ & Quercetin & $?$ & $?$ & $?$ \\
\hline $\begin{array}{l}\text { Zheng et al. } \\
2017 \text { [35] }\end{array}$ & Quercetin & China Institute of Food and Drug Verification & $(\geq 98 \%)$ & $\begin{array}{l}\text { Batch number: } \\
100081201509\end{array}$ \\
\hline $\begin{array}{l}\text { Abdelkarem et al. } \\
2016[36]\end{array}$ & Quercetin & Sigma-Aldrich Corporation, USA & $(\geq 99 \%)$ & HPLC \\
\hline $\begin{array}{l}\text { Bian et al. } \\
2016[37]\end{array}$ & Quercetin & Ai Ke Da Chemical Reagent Co., Ltd., CHN & $?$ & HPLC \\
\hline $\begin{array}{l}\text { Feng et al. } \\
2016[38]\end{array}$ & Quercetin & $\begin{array}{l}\text { Institute of occupational health and occupational disease, } \\
\text { Chinese Academy of Preventive Medicine, CHN }\end{array}$ & $?$ & Batch number: 911015 \\
\hline $\begin{array}{l}\text { Zhou et al. } \\
2016 \text { [39] }\end{array}$ & Quercetin & Sigma-Aldrich Corporation, USA & $(\geq 99 \%)$ & HPLC \\
\hline $\begin{array}{l}\text { Tian et al. } \\
2016[40]\end{array}$ & Quercetin & Sigma-Aldrich Corporation, USA & $(\geq 99 \%)$ & HPLC \\
\hline $\begin{array}{l}\text { Derakhshanian } \\
\text { et al. } 2012[41]\end{array}$ & Quercetin & Sigma-Aldrich Corporation, USA & $95 \%$ & HPLC \\
\hline $\begin{array}{l}\text { Liang et al. } \\
2011[42]\end{array}$ & Quercetin & Sigma-Aldrich Corporation, USA & $?$ & HPLC \\
\hline $\begin{array}{l}\text { Siddiqui et al. } \\
2011[27]\end{array}$ & Quercetin & Sigma-Aldrich Corporation, USA & $?$ & HPLC \\
\hline $\begin{array}{l}\text { Siddiqui et al. } \\
2011 \text { [27] }\end{array}$ & $\begin{array}{l}\text { Quercetin-6-C-A-D- } \\
\text { glucopyranoside }\end{array}$ & Purificated by themself & $?$ & HPLC \\
\hline $\begin{array}{l}\text { Tsuji et al. } \\
2009 \text { [43] }\end{array}$ & Quercetin & Sigma-Aldrich Corporation, USA & $?$ & HPLC \\
\hline $\begin{array}{l}\text { Wang et al. } \\
2008[44]\end{array}$ & Quercetin & Shaanxi Huike Biology Co., Ltd., CHN & $?$ & $?$ \\
\hline $\begin{array}{l}\text { Zhu and Wei } \\
2005[45]\end{array}$ & Quercetin & $\begin{array}{c}\text { Products of labor and health institution, Chinese } \\
\text { Academy of Preventive Medicine, CHN }\end{array}$ & $?$ & Batch number: 911015 \\
\hline Marie 2000 & $\begin{array}{l}\text { Quercetin-3-O- } \\
\text { rutinose }\end{array}$ & Sigma-Aldrich Corporation, USA & $?$ & HPLC \\
\hline
\end{tabular}

HPLC: high-performance liquid chromatography.

SMD 1.98, 95\% CI (1.67, 2.29), $P<0.00001$; heterogeneity: $\chi^{2}=59.44, I^{2}=76 \%$, Figure 3$)$ and L-BMD $(n=50$, SMD 3.96, 95\% CI $(2.91,5.01), P<0.00001$; heterogeneity: $\chi^{2}=$ $0.27, I^{2}=0 \%$, Figure 4) compared with the control group. Under micro-CT, meta-analysis of 6 researches $[27,30,33$, $43]$ and 6 researches $[27,30,33,43]$ separately showed a sig- nificant effect of $\mathrm{Q}$ or its derivatives for increasing Tb.Th $(n=106$, SMD $0.96,95 \%$ CI $(0.49,1.43), P<0.00001$; heterogeneity: $\chi^{2}=41.74, I^{2}=88 \%$, Figure $\left.5(\mathrm{a})\right)$ and Tb.N $(n=106$, SMD 2.08, 95\% CI (1.47, 2.68), $P<0.00001$; heterogeneity: $\chi^{2}=54.43, I^{2}=91 \%$, Figure 5(b)). About physical mechanics index, meta-analysis of 9 researches $[27,32,34,37,39-42$, 
TABLE 3: Risk of bias of the included studies.

\begin{tabular}{|c|c|c|c|c|c|c|c|c|c|c|c|}
\hline Study & A & $\mathrm{B}$ & $\mathrm{C}$ & $\mathrm{D}$ & $\mathrm{E}$ & $\mathrm{F}$ & G & $\mathrm{H}$ & $\mathrm{I}$ & $\mathrm{J}$ & Total \\
\hline Geng et al. 2019 [4] & $\sqrt{ }$ & $\sqrt{ }$ & $\sqrt{ }$ & $\sqrt{ }$ & & $\sqrt{ }$ & & & $\sqrt{ }$ & & 6 \\
\hline Min et al. 2019 [30] & & $\sqrt{ }$ & $\sqrt{ }$ & & & $\sqrt{ }$ & & & $\sqrt{ }$ & & 4 \\
\hline Nada et al. 2018 [31] & $\sqrt{ }$ & $\sqrt{ }$ & & $\sqrt{ }$ & & $\sqrt{ }$ & & & $\sqrt{ }$ & $\sqrt{ }$ & 6 \\
\hline Yuan et al. 2018 [32] & $\sqrt{ }$ & $\sqrt{ }$ & $\sqrt{ }$ & & & & & & $\sqrt{ }$ & $\sqrt{ }$ & 5 \\
\hline Yuan et al. 2018 [33] & $\sqrt{ }$ & & $\sqrt{ }$ & & & & & & $\sqrt{ }$ & $\sqrt{ }$ & 4 \\
\hline Xing et al. 2017 [34] & $\sqrt{ }$ & $\sqrt{ }$ & $\sqrt{ }$ & & & $\sqrt{ }$ & & & $\sqrt{ }$ & & 5 \\
\hline Zheng et al. 2017 [35] & $\sqrt{ }$ & $\sqrt{ }$ & $\sqrt{ }$ & $\sqrt{ }$ & & $\sqrt{ }$ & & & $\sqrt{ }$ & $\sqrt{ }$ & 7 \\
\hline Abdelkarem et al. 2016 [36] & $\sqrt{ }$ & $\sqrt{ }$ & $\sqrt{ }$ & $\sqrt{ }$ & & $\sqrt{ }$ & & & $\sqrt{ }$ & $\sqrt{ }$ & 7 \\
\hline Bian et al. 2016 [37] & $\sqrt{ }$ & $\sqrt{ }$ & $\sqrt{ }$ & & $?$ & $\sqrt{ }$ & & & $\sqrt{ }$ & $\sqrt{ }$ & 6 \\
\hline Feng et al. 2016 [38] & $\sqrt{ }$ & $\sqrt{ }$ & $\sqrt{ }$ & & & $\sqrt{ }$ & & & & $\sqrt{ }$ & 5 \\
\hline Zhou et al. 2016 [39] & & $\sqrt{ }$ & $\sqrt{ }$ & & & $\sqrt{ }$ & & & $\sqrt{ }$ & & 4 \\
\hline Tian et al. $2014[40]$ & $\sqrt{ }$ & $\sqrt{ }$ & $\sqrt{ }$ & & & & & & $\sqrt{ }$ & $\sqrt{ }$ & 5 \\
\hline Derakhshanian et al. 2013 [41] & $\sqrt{ }$ & $\sqrt{ }$ & $\sqrt{ }$ & & & $\sqrt{ }$ & & & $\sqrt{ }$ & $\sqrt{ }$ & 6 \\
\hline Liang et al. $2011[42]$ & $\sqrt{ }$ & $\sqrt{ }$ & $\sqrt{ }$ & & & $\sqrt{ }$ & & & & $\sqrt{ }$ & 5 \\
\hline Siddiqui et al. 2011 [27] & $\sqrt{ }$ & $\sqrt{ }$ & $\sqrt{ }$ & & & & & & & $\sqrt{ }$ & 4 \\
\hline Tsuji et al. 2009 [43] & $\sqrt{ }$ & $\sqrt{ }$ & $\sqrt{ }$ & $\sqrt{ }$ & & $?$ & & & $\sqrt{ }$ & $\sqrt{ }$ & 6 \\
\hline Wang et al. 2008 [44] & $\sqrt{ }$ & & $\sqrt{ }$ & & & $\sqrt{ }$ & & & $\sqrt{ }$ & & 4 \\
\hline Zhu and Wei 2005 [45] & $\sqrt{ }$ & & $\sqrt{ }$ & & & & & & $\sqrt{ }$ & & 3 \\
\hline Marie 2000 & $\sqrt{ }$ & $\sqrt{ }$ & & & & $\sqrt{ }$ & & & $\sqrt{ }$ & & 4 \\
\hline
\end{tabular}

Note: studies fulfilling the criteria of: A: peer-reviewed publication; B: control of temperature; C: random allocation to treatment or control; D: blinded induction of model (group randomly after modeling); E: blinded assessment of outcome; F: use of anesthetic without significant protective and toxic effects on bones; G: appropriate animal model (aged, hyperlipemia, hypertensive, or diabetes); H: sample size calculation; I: compliance with animal welfare regulations (including three or more of the following points: preoperative anaesthesia, postoperative analgesia, nutrition, disinfection, environment temperature, environment humidity, circadian rhythm, and euthanasia); J: statement of potential conflict of interests.

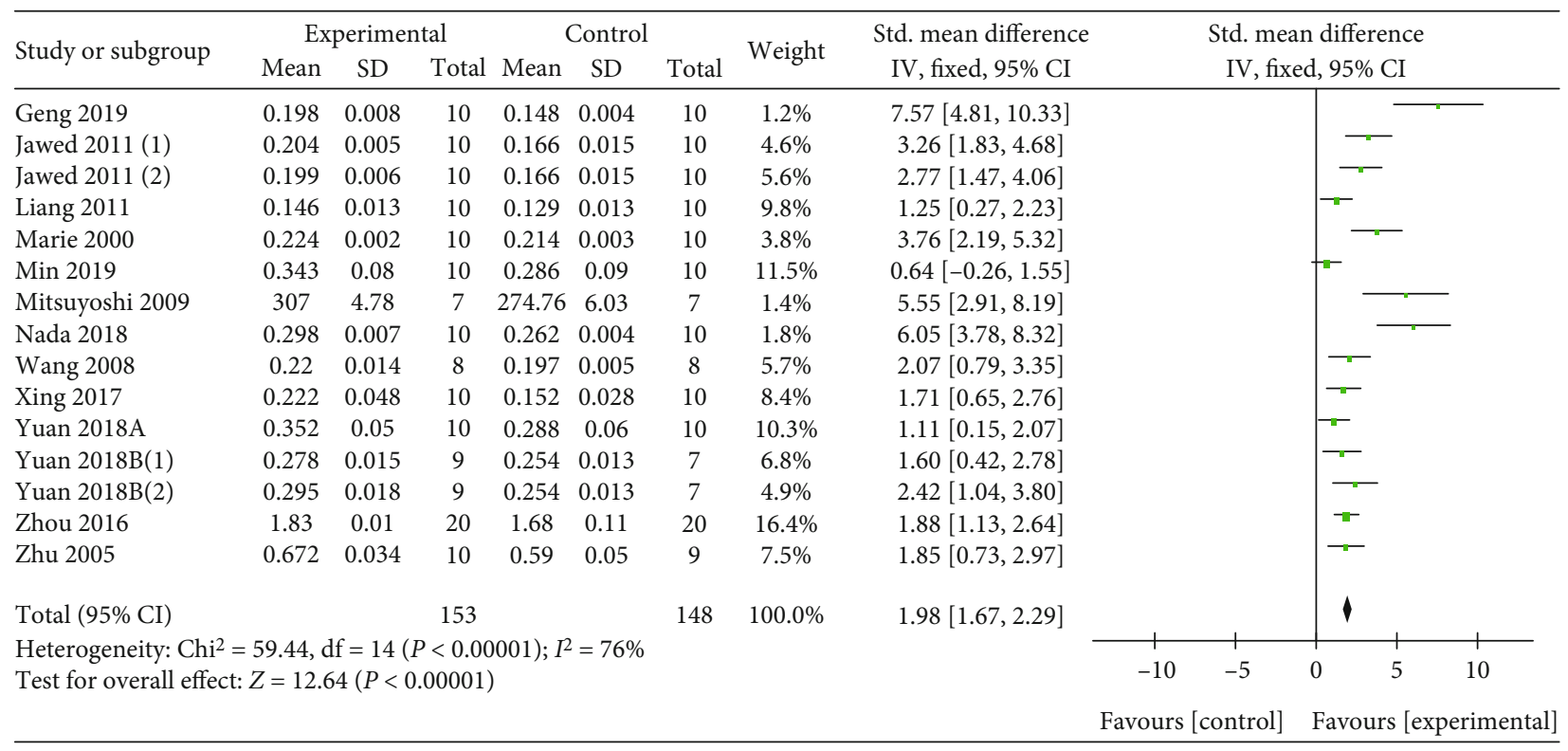

FIgURE 3: The forest plot: effects of Q or its derivatives for increasing L-BMD compared with the control group.

45] showed a significant effect of $Q$ or its derivatives for increasing bone maximum load $(n=216$, SMD 1.33, 95\% CI $(1.0,1.66), P<0.00001$; heterogeneity: $\chi^{2}=63.69, I^{2}=$ $87 \%$, Figure 6$)$. In consideration of high heterogeneity, sensitivity analyses of the above indicators were carried out, and the result showed that the heterogeneity did not substantially alter after removing any 1 study.

3.4.3. Serum ALP, OC, and CTX. Compared with the control group, $\mathrm{Q}$ and its derivatives were reported that they existed 


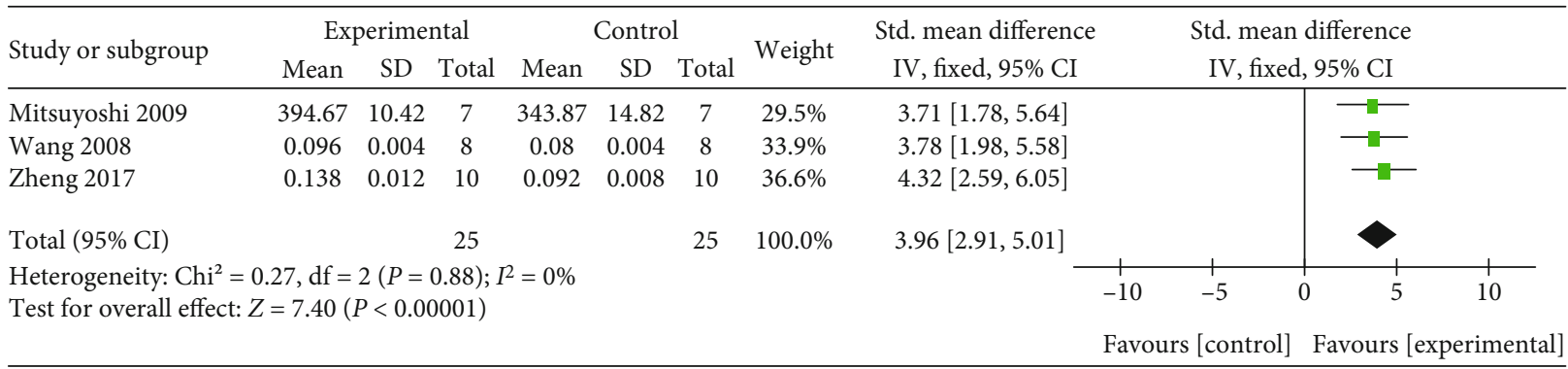

FIGURE 4: The forest plot: effects of Q or its derivatives for increasing F-BMD compared with the control group.

\begin{tabular}{|c|c|c|c|c|c|c|c|c|c|c|}
\hline \multirow{3}{*}{$\begin{array}{l}\text { Study or subgroup } \\
\text { Jawed } 2011(1)\end{array}$} & \multicolumn{3}{|c|}{ Experimental } & \multicolumn{3}{|c|}{ Control } & \multirow{3}{*}{$\begin{array}{c}\text { Weight } \\
7.6 \%\end{array}$} & \multirow{3}{*}{$\begin{array}{c}\text { Std. mean difference } \\
\text { IV, fixed, 95\% CI } \\
4.24[2.53,5.95]\end{array}$} & \multirow{2}{*}{\multicolumn{2}{|c|}{$\begin{array}{c}\text { Std. mean difference } \\
\text { IV, fixed, 95\% CI }\end{array}$}} \\
\hline & \multirow{2}{*}{$\frac{\text { Mean }}{96.8}$} & \multirow{2}{*}{$\frac{\mathrm{SD}}{4}$} & \multirow{2}{*}{$\frac{\text { Total }}{10}$} & \multirow{2}{*}{$\frac{\text { Mean }}{79.3}$} & \multirow{2}{*}{$\begin{array}{c}\mathrm{SD} \\
3.9\end{array}$} & \multirow{2}{*}{$\frac{\text { Total }}{10}$} & & & & \\
\hline & & & & & & & & & \multirow{3}{*}{$\rightarrow$} & \multirow[t]{2}{*}{$\square$} \\
\hline Jawed 2011 (2) & 74.3 & 5.2 & 10 & 79.3 & 3.9 & 10 & $24.8 \%$ & $-1.04[-1.99,-0.09]$ & & \\
\hline Min 2019 & 0.134 & 0.018 & 10 & 0.104 & 0.023 & 10 & $22.3 \%$ & $1.39[0.39,2.39]$ & & - \\
\hline Mitsuyoshi 2009 & 27.729 & 10 & 7 & 25.913 & 1.475 & 7 & $20.1 \%$ & $0.24[-0.81,1.29]$ & & \multirow{2}{*}{-} \\
\hline Yuan 2018B(1) & 0.2 & 0.03 & 9 & 0.14 & 0.04 & 7 & $15.9 \%$ & $1.64[0.45,2.82]$ & & \\
\hline Yuan $2018 \mathrm{~B}(2)$ & 0.25 & 0.03 & 9 & 0.14 & 0.04 & 7 & $9.2 \%$ & $3.00[1.45,4.55]$ & & \\
\hline \multicolumn{3}{|l|}{ Total $(95 \% \mathrm{CI})$} & \multicolumn{3}{|l|}{55} & 51 & $100.0 \%$ & $0.96[0.49,1.43]$ & & \\
\hline \multicolumn{9}{|c|}{ Heterogeneity: $\mathrm{Chi}^{2}=41.74, \mathrm{df}=5(P<0.00001) ; I^{2}=88 \%$} & $\begin{array}{ll}-4 & -2\end{array}$ & 24 \\
\hline \multicolumn{9}{|c|}{ Test for overall effect: $Z=3.99(P<0.0001)$} & Favours [control] & Favours [experimental] \\
\hline
\end{tabular}

(a)

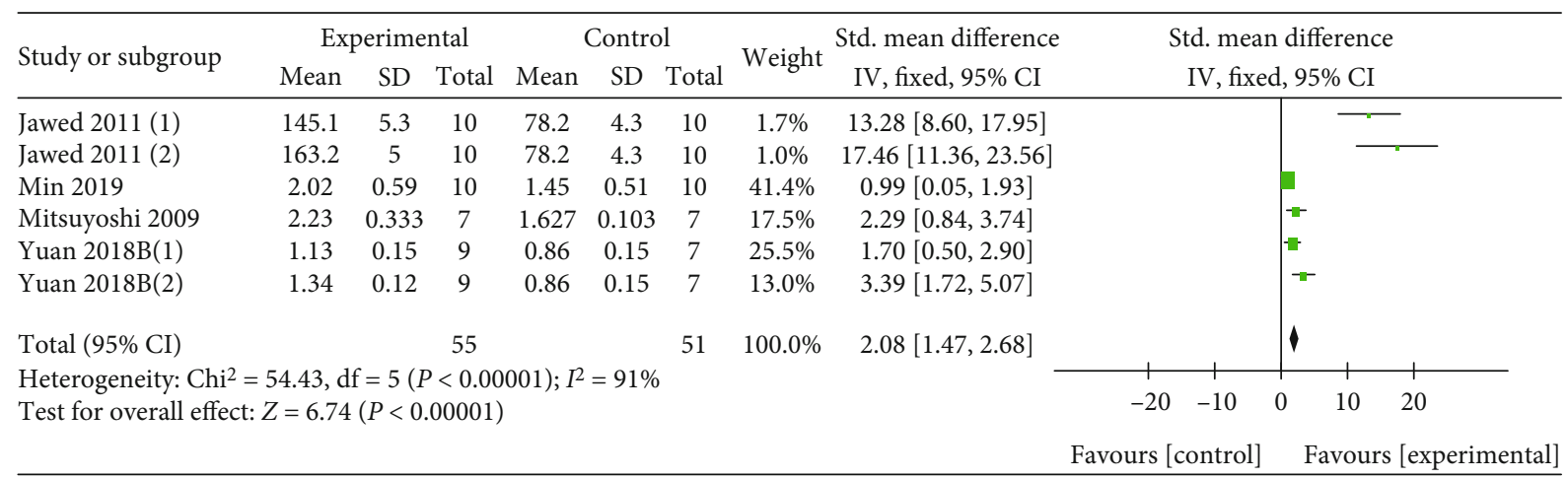

(b)

FIgURE 5: (a) The forest plot: effects of Q or its derivatives for increasing Tb.Th compared with the control group; (b) The forest plot: effects of $\mathrm{Q}$ or its derivatives for increasing Tb.N compared with the control group.

significant effect for increasing serum ALP $(P<0.05)$ in 7 studies [30, 33-36, 42], no significant effect for serum ALP $(P>0.05)$ in 1 study [31], and significant effect for reducing serum ALP $(P<0.05)$ in 1 study [45]. Besides, 5 studies $[30,35,37,41,42]$ and 3 studies $[34,35,41]$ reported separately that $\mathrm{Q}$ could increase the serum OC $(P<0.05)$ and/or reduce the serum CTX $(P<0.05)$.

3.4.4. Serum Estradiol and Uterine Weight. Q and its derivatives were found to increase serum estradiol level relatively $(n=60, \mathrm{SMD} 1.03,95 \% \mathrm{CI}(0.41,1.65), P<0.00001$; heterogeneity: $\chi^{2}=21.95, I^{2}=91 \%$, Figure 7$)$. As a result of estradiol effects, the uterine weight of experimental animals was slightly increased compared with the control group $(n=86$, SMD 1.31, 95\% CI $(0.80,1.82), P<0.00001$; heterogeneity: $\chi^{2}=13.91, I^{2}=71 \%$, Figure 8$)$.

3.4.5. Indicators of Adverse Reactions. Four studies [4, 33, 38, 45] utilized survival rate as primary outcome measure, and meta-analysis of 3 studies showed no statistical difference of $\mathrm{Q}$ or its derivatives on survival rate $(n=94, \mathrm{RR} 1.00,95 \%$ CI $(0.89,1.13), P=0.94$; heterogeneity: $\chi^{2}=2.34, I^{2}=0 \%$, Figure 9). BUN was measured in 2 studies $[31,34]$ and $\mathrm{SCr}$ in 1 study [34] to assess the adverse effect of Q to the renal function. In addition, AST and ALT were measured in 1 study [31] to assess the adverse effect of Q to the liver, and 


\begin{tabular}{|c|c|c|c|c|c|c|c|c|c|c|c|}
\hline \multirow{2}{*}{ Study or subgroup } & \multicolumn{3}{|c|}{ Experimental } & \multicolumn{3}{|c|}{ Control } & \multirow{2}{*}{ Weight } & \multirow{2}{*}{$\begin{array}{l}\text { Std. mean difference } \\
\text { IV, fixed, 95\% CI }\end{array}$} & \multirow{2}{*}{\multicolumn{3}{|c|}{$\begin{array}{l}\text { Std. mean difference } \\
\text { IV, fixed, 95\% CI }\end{array}$}} \\
\hline & Mean & SD & Total & Mean & $\mathrm{SD}$ & Total & & & & & \\
\hline Bian 2016 & 81 & 14.4 & 10 & 77.4 & 11.6 & 10 & $14.2 \%$ & $0.26[-0.62,1.14]$ & & - & \\
\hline Hoda 2012 & 112 & 10.67 & 8 & 79.56 & 11.56 & 8 & $5.1 \%$ & $2.76[1.28,4.23]$ & & & \\
\hline Jawed 2011 (2) & 112.3 & 3.2 & 10 & 88.57 & 4.2 & 10 & $2.1 \%$ & $6.09[3.81,8.37]$ & & & \\
\hline Liang 2011 & 165.81 & 11.5 & 10 & 115.97 & 14.38 & 10 & $4.6 \%$ & $3.67[2.12,5.21]$ & & & \\
\hline Tian 2014 & 19.24 & 1.33 & 20 & 17.36 & 2.04 & 20 & $24.7 \%$ & $1.07[0.40,1.74]$ & & - & \\
\hline Xing 2017 & 91.49 & 14.32 & 10 & 51.66 & 6.85 & 10 & $5.1 \%$ & $3.40[1.93,4.86]$ & & & \\
\hline Yuan 2018A & 88.5 & 7.76 & 10 & 79.84 & 3.65 & 10 & $11.1 \%$ & $1.37[0.37,2.36]$ & & & \\
\hline Zhou 2016 & 16.05 & 0.96 & 20 & 13.91 & 1.34 & 20 & $19.8 \%$ & $1.80[1.05,2.55]$ & & & \\
\hline Zhu 2005 & 91.9 & 16.5 & 10 & 103 & 13.7 & 10 & $13.3 \%$ & $-0.70[-1.61,0.21]$ & & & \\
\hline \multicolumn{3}{|l|}{ Total $(95 \% \mathrm{CI})$} & 108 & & & 108 & $100.0 \%$ & $1.33[1.00,1.66]$ & & & \\
\hline \multicolumn{9}{|c|}{ 1est ior overall entect. $2-1.03(1<0.00001)$} & Favours [control] & Favours & [experimental] \\
\hline
\end{tabular}

FIGURE 6: The forest plot: effects of Q or its derivatives for increasing bone maximum load compared with the control group.

\begin{tabular}{|c|c|c|c|c|c|c|c|c|c|}
\hline \multirow{2}{*}{ Study or subgroup } & \multicolumn{3}{|c|}{ Experimental } & \multicolumn{3}{|c|}{ Control } & \multirow{2}{*}{ Weight } & \multirow{2}{*}{$\begin{array}{l}\text { Std. mean difference } \\
\text { IV, fixed, } 95 \% \text { CI }\end{array}$} & \multirow{2}{*}{$\begin{array}{l}\text { Std. mean difference } \\
\text { IV, fixed, } 95 \% \text { CI }\end{array}$} \\
\hline & Mean & SD & Total & Mean & $\mathrm{SD}$ & Total & & & \\
\hline Bian 2016 & 29.2 & 1.99 & 10 & 28 & 1.77 & 10 & $47.5 \%$ & $0.61[-0.29,1.51]$ & \\
\hline Zheng 2017 & 31.4 & 3 & 10 & 12.5 & 2.1 & 10 & $5.8 \%$ & $6.99[4.42,9.56]$ & \\
\hline Zhu 2005 & 32.9 & 20.6 & 10 & 21.5 & 7.1 & 10 & $46.6 \%$ & $0.71[-0.20,1.62]$ & \\
\hline Total $(95 \%$ CI $)$ & & & 30 & & & 30 & $100.0 \%$ & $1.03[0.41,1.65]$ & \\
\hline \multicolumn{9}{|c|}{$\begin{array}{l}\text { Heterogeneity: } \mathrm{Chi}^{2}=21.95, \mathrm{df}=2(P<0.0001) ; I^{2}=91 \% \\
\text { Test for overall effect: } Z=3.24(P=0.001)\end{array}$} & $\begin{array}{llllll}-4 & -2 & 0 & 2 & 4\end{array}$ \\
\hline & & & & & & & & & Favours [experimental] \\
\hline
\end{tabular}

Figure 7: The forest plot: effects of $Q$ or its derivatives for increasing serum estradiol level compared with the control group.

the results showed that there was no statistical difference in renal and liver function between $\mathrm{Q}$ group and control group $(P>0.05)$.

3.5. Subgroup Analysis. F-BMD was reported to be improved greatly in 15 comparisons [4, 27, 30-34, 39, 42-46]. The potential confounding factors which may increase the heterogeneity of F-BMD were explored via subgroup analysis. First, we divide the 15 comparisons into the ovariectomized model group and nonovariectomized model group according to different modeling methods. As the result, no significant difference was observed in the effect size of two groups $(\mathrm{SMD}=2.00 \pm 0.36$ versus $\mathrm{SMD}=1.93 \pm 0.58, \quad P=0.85$, Figure 10(a)) and heterogeneity of both groups did not decrease obviously. On the basis of the result of the subgroup analysis above, we analyzed the F-BMD in different subgroups stratified according to the following variables in the ovariectomized model group: different animal species, different laboratory drugs, and the duration of treatment. In the subgroup analysis of these factors, the mice group showed better effect size than the rat group $(\mathrm{SMD}=5.55 \pm 2.64$ versus $\mathrm{SMD}=1.93 \pm 0.37, P=0.008$, Figure $10(\mathrm{~b})$ ) with significantly reduced heterogeneity of both groups. Another subgroup analysis indicated that the effect of $\mathrm{Q}$ derivatives was better than $Q$ with better evaluation effect size
$(\mathrm{SMD}=3.28 \pm 0.78$ versus $\mathrm{SMD}=1.64 \pm 0.41, P=0.0003$, Figure $10(\mathrm{c})$ ), and the heterogeneity experienced a marked decline in $\mathrm{Q}$ derivatives treatment group. In addition, the shorter period of Q or its derivatives treatment showed batter effect size than the longer treatment $(\mathrm{SMD}=5.55 \pm 2.64$ versus $\mathrm{SMD}=1.69 \pm 0.43$ versus $\mathrm{SMD}=2.16 \pm 0.64, P=0.01$, Figure 10(d)).

\section{Discussion}

4.1. Summary of Evidence. The first-ever preclinical systematic review included a batch of studies of acceptable quality to estimate the efficacy and mechanisms of $\mathrm{Q}$ and its derivatives in animal models of osteoporosis. The findings revealed $\mathrm{Q}$ and its derivatives are potential antiosteoporosis drug via multiple mechanisms.

4.2. Limitations. Some limitations of the meta-analysis and the system evaluation were listed as follows: (1) there may still be a certain degree of selective bias due to the lack of negative studies and the studies from other databases or in other languages; (2) defects in aspects of blinding assessment of outcome and sample size calculation may affect the accuracy of findings [47]; (3) the few number of studies modeled by nonovariectomized methods leads to that only comparisons 


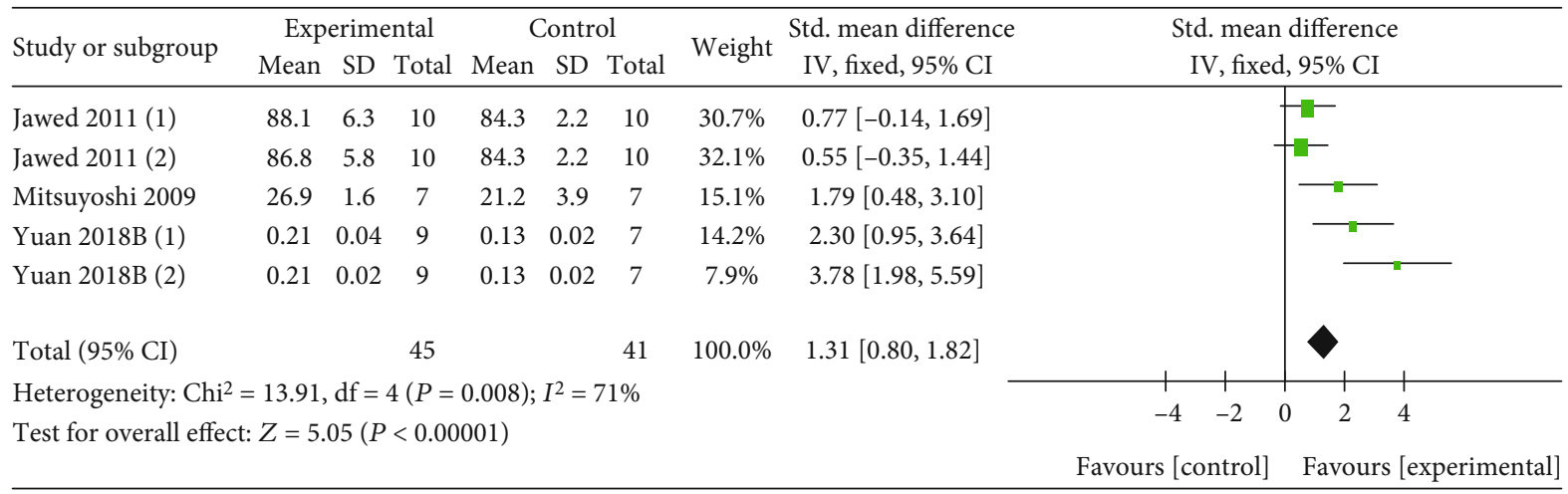

FIGURE 8: The forest plot: effects of Q or its derivatives for increasing uterine weight of experimental animals compared with the control group.

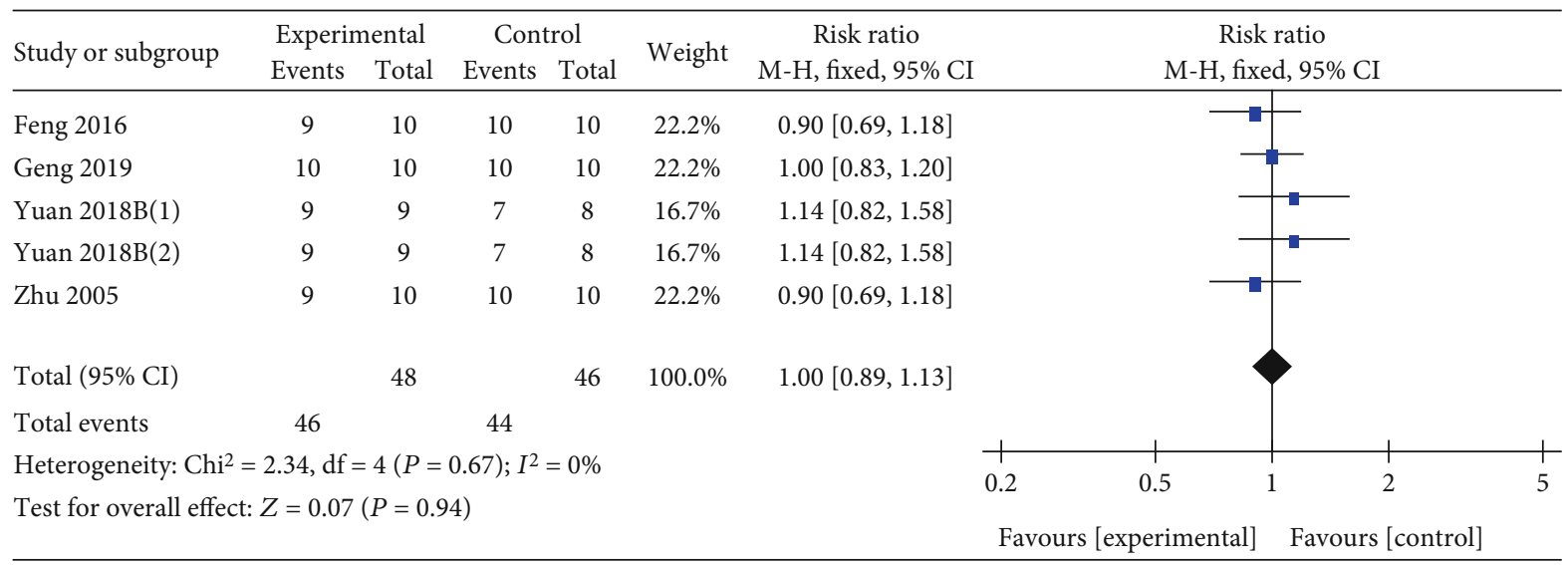

FIGURE 9: The forest plot: effects of $Q$ or its derivatives on the survival rate of experimental animals compared with the control group.

between groups are carried out rather than systematic evaluations in those studies; (4) no study utilized animals with relevant complication.

\subsection{Implication}

4.3.1. Animal Model Selection. Using different animal models at different research stages of disease is crucial to study it pathophysiology and treatments [48]. Factors that need to be considered include pathogenesis of model, availability of the animals, technical requirements, and cost and ethical considerations [49]. According to the pathogenesis, animal models of osteoporosis can be divided into two types: models with increased bone resorption as the dominant mechanism (such as ovariectomized osteoporosis model, disused osteoporosis model, retinoic acid induction model, nutritional osteoporosis model, and glucocorticoid model) and models with reduced bone formation as the dominant mechanism (such as senile osteoporosis model and $\mathrm{n}-\mathrm{ZnO}$ induction model) [50]. The present study comprehensively contains the ovariectomized osteoporosis model, retinoic acid or n$\mathrm{ZnO}$ induction model, diabetic osteoporosis, and glucocorticoid model to estimate the efficacy and mechanisms of Q and its derivatives for osteoporosis. And the results of metaanalysis and subgroup analysis reflected that $\mathrm{Q}$ and its deriv- atives could play a role in both two mechanisms of osteoporosis $(\mathrm{SMD}=2.00 \pm 0.36$ versus $\mathrm{SMD}=1.93 \pm 0.58, P=0.85$, Figure 10(a)). However, some key points to establish incorporating models still deserve attention. The ovariectomized rats, as the best recognized postmenopausal osteoporosis model, are the most adopted model in eligible studies. Animals with mature skeleton are obligatory for osteoporosis researches [51]. In the present study, some immature rats ( $<12$ weeks) whose bone mass was below its peak [32, 33, 43] were used to establish models, causing that the confounding factors are introduced to animals that are still accruing bone [51]. Thus, the application of animals with mature bone needs to be emphasized for future osteoporosis experiments. In addition, the dosage of glucocorticoids should be grasped well to avoid no change in bone mass in low dosage or animal death due to overimmunization in high dosage. Preexperiment as Derakhshanian et al. [41] did to explore the appropriate dosage is a recommended practice.

The subgroup analysis showed better effect size in the mice group than the rat group $(\mathrm{SMD}=5.55 \pm 2.64$ versus $\mathrm{SMD}=1.93 \pm 0.37, P=0.008$, Figure $10(\mathrm{~b}))$ with significantly reduced heterogeneity of both groups, suggesting that different animals may be one of the main sources of heterogeneity. The commonly used animals in osteoporosis experiments are rodent, rabbit, dog, sheep, primates, and so on 


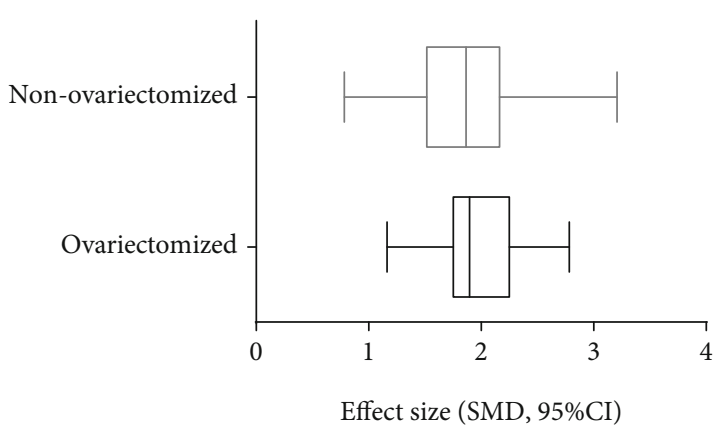

(a)

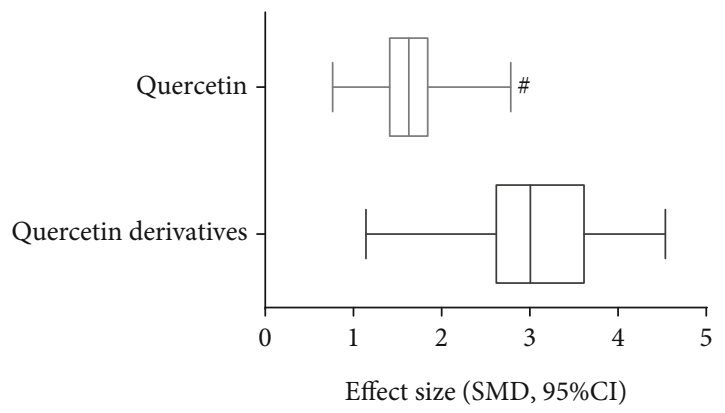

(c)

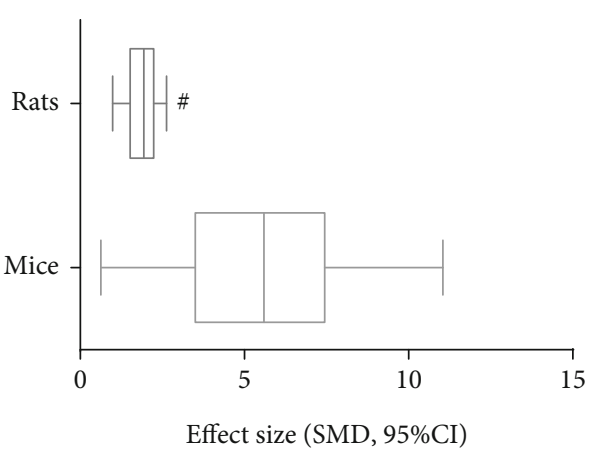

(b)

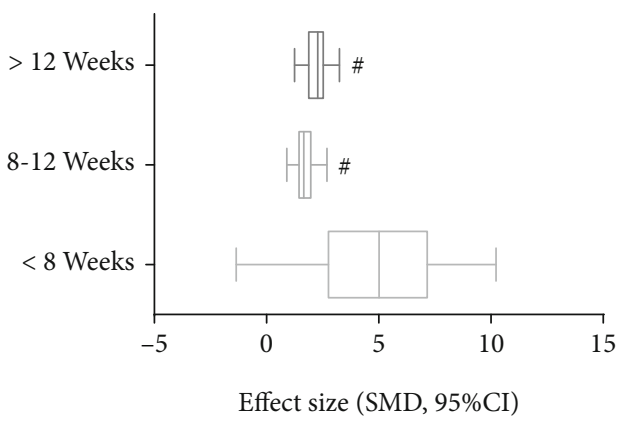

(d)

FIGURE 10: Subgroup analyses of the F-BMD. (a) The different effect size between the ovariectomized model group and nonovariectomized model group; (b) the different effect size between mice and rats; (c) the different effect size between $Q$ and its derivatives; (d) the different effect size between different treatment time group. ${ }^{\#} P<0.05$ vs. control groups; ${ }^{*} P>0.05$ vs. control groups.

$[52,53]$. Rodents such as rats and mice possess the advantages of being repeatable, cheap, and convenient to be bred and anesthetized. It also takes less time to form a new balance of bone remodeling. After ovariectomy in rodents, the bone mass of cancellous bone decrease and the bone turnover rate increase, which resembles to that of osteoporosis in postmenopausal women and estrogen replacement therapy could alleviate bone mass loss [54]. Therefore, female rodents are widely used in the study of postmenopausal osteoporosis. It was regarded as a preferred animal for small- or mediumsized laboratories to investigate the efficacy and mechanisms of drugs. However, it is not suitable for the study of the bone cortex because of the absence of haversian system in the bone cortex of rats or mice. Additionally, difficulties arose when the arm of the study was to implant fixation or prosthetic devices as well as that studies which need several the collection of high blood volumes or surgical operation or several biopsies due to its small size especially in mice [55]. Compared to rodents, rabbits and dogs have the convenience of appropriate cost in terms of purchase and maintenance and reasonable anatomical size which was advantageous to biopsies, blood collection repeatedly, and surgical treatments such as bone implant and bone-implant interface. On the other hand, it is suitable for the study of the effect of cortical bone because of the obvious Harvard reconstruction. However, no significant change was observed in bone mineral density after ovariectomy in rabbits and dogs [56]. The combination of surgery and subsequent glucocorticoid treatment is the best solution at present to obtain bone mineral reduction consistently in the short term in rabbits [48]. However, it cannot be achieved in dogs now which may be related to the low estrogen level in female dogs. Apart from the advantages of rabbits and dogs, sheep are ideal models for the study of vertebral osteoporosis [57] and they also have similar toxic effects on osteocytes by fluoride to humans [58]. Unfortunately, they are not suitable to be adopted to study the efficacy of oral administration of drugs given that the sheep are ruminants. Additionally, bone mineral density, blood biochemical parameters, and bone histomorphology of sheep all fluctuated seasonally [59] which may influence the accuracy of experimental results. From both a physiological and anatomical standpoint, the characteristics of the skeleton of primates are most close to humans than any other type of animal. Nevertheless, obtaining licenses to use them as experimental animal become increasingly difficult due to ethical considerations [60] and potential epidemic animal-borne diseases in primates [61]. Meanwhile, the high cost to purchase and maintain primates restricts their use in experimentation [49]. In consideration of that, the arm of the present studies is to explore the preliminary effect of and mechanisms of Q in animal models of osteoporosis, thus the use of rodents is acceptable at this stage. We suggest the advanced animals (sheep, primates, etc.) or animal models (transgenic or knockout rodents, etc.) for osteoporosis should be chosen in the future on the basis of the experimental purpose and permissible conditions.

4.3.2. Other Subgroup Analyses. A better evaluation effect size in a group of $\mathrm{Q}$ derivatives $(\mathrm{SMD}=3.28 \pm 0.78$ versus $\mathrm{SMD}$ $=1.64 \pm 0.41, P=0.0003$, Figure $10(\mathrm{c}))$ was indicated in 
TABle 4: The derivatives of $\mathrm{Q}$.

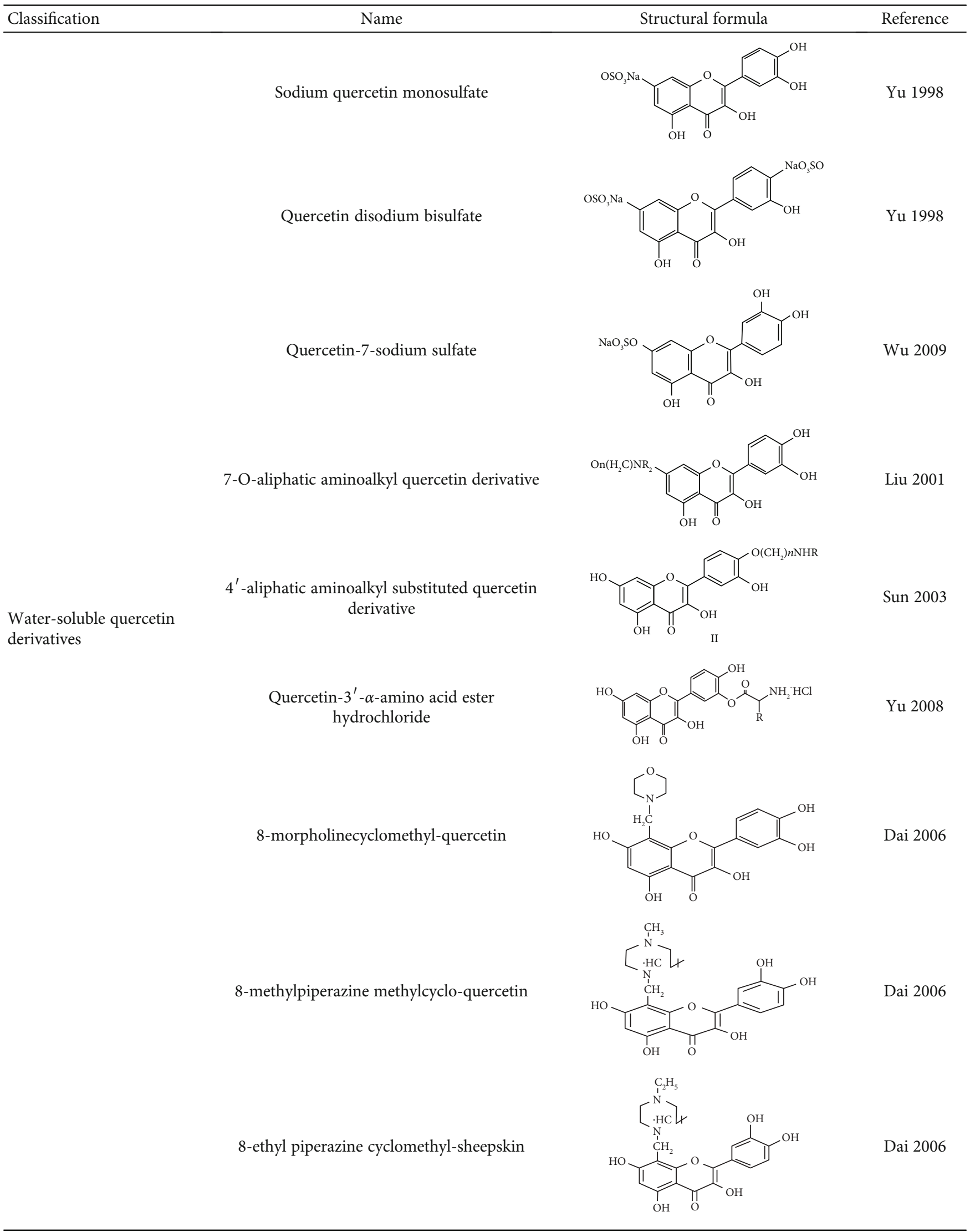


TABle 4: Continued.

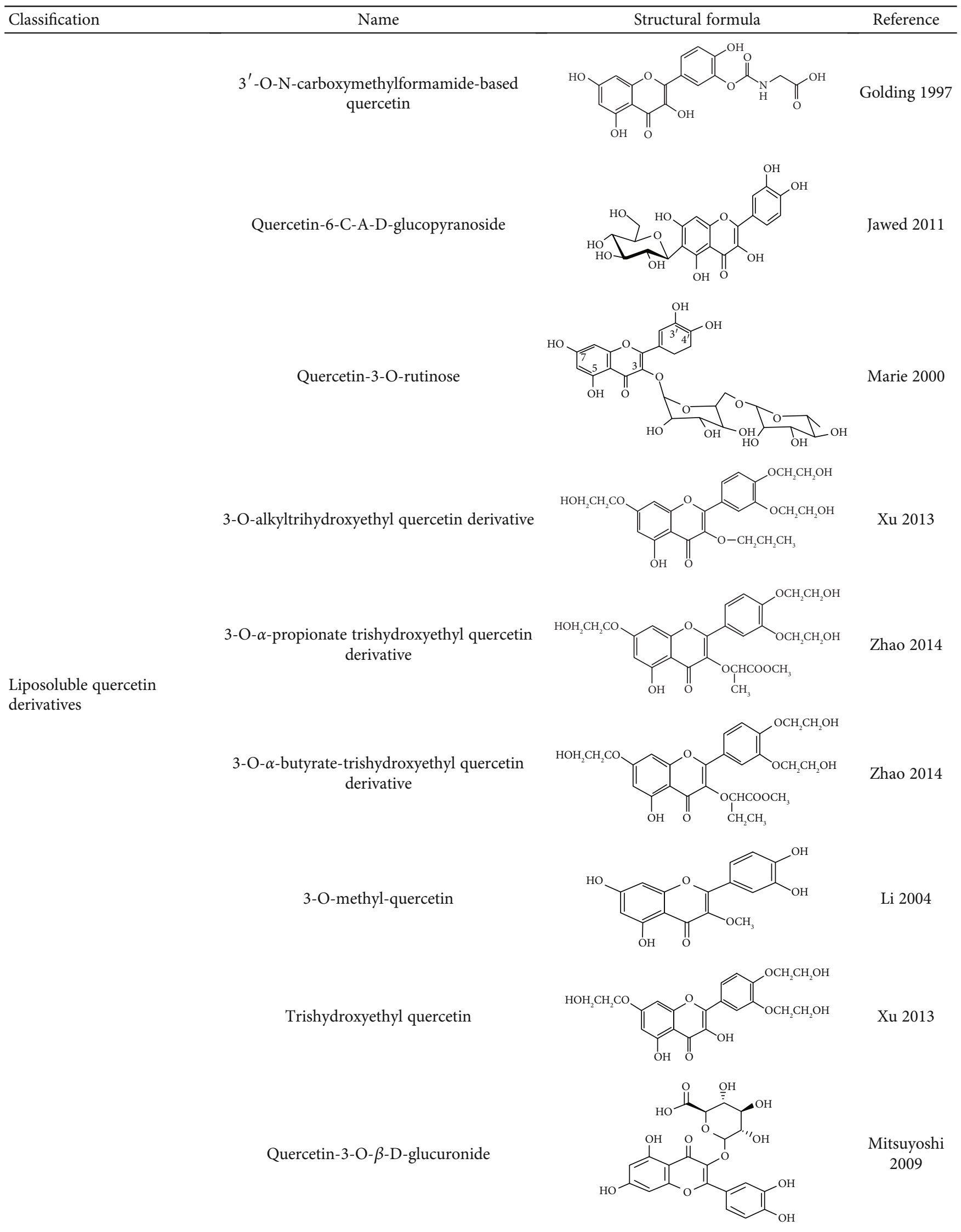




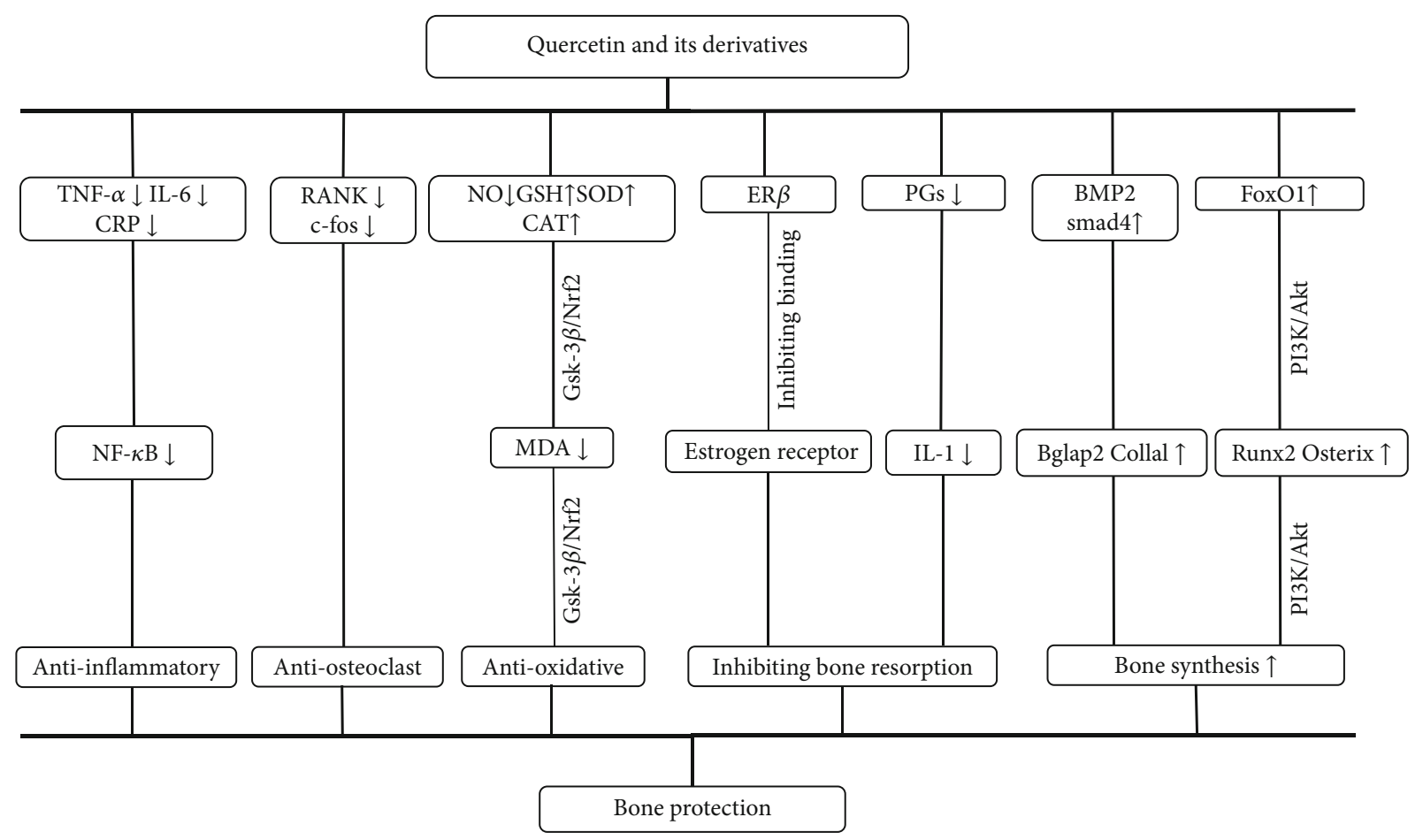

FIGURE 11: A schematic representation of osteoprotective mechanisms of Q and its derivatives for osteoporosis.

subgroup analysis based on contrast of the overall effect in different experiments. Given the existence of mixed factors in different experiments, all included studies have been perused again for these studies were designed to contrast the different therapeutic effects between $\mathrm{Q}$ and its derivatives under the same experimental conditions. Among, QCG (quercetin-6-C-A-D-glucopyranoside) [27] and rutin (quercetin-3-O-B-rutinoside) [33] were reported to improve bone biomechanical quality more effectively than $\mathrm{Q}$ via positive modifications of bone microarchitecture and bone mineral density without hyperplastic effect on the uterus, which possibly is related to that the synthesis of modified groups attached to Q improves solubility and bioavailability [62]. In this context, all the existing derivatives of $\mathrm{Q}$ were collected and listed in Table 4, which are recommended as potential antiosteoporosis drugs in the future researches. Another subgroup analysis showed the shorter period of $\mathrm{Q}$ or its derivative treatment showed better effect size than the longer treatment $(\mathrm{SMD}=5.55 \pm 2.64$ versus $\mathrm{SMD}=1.69 \pm 0.43$ versus $\mathrm{SMD}=2.16 \pm 0.64, P=0.01$, Figure $10(\mathrm{~d}))$, suggesting that the duration of treatment may be a source of high heterogeneity. For the reason, we attribute it to that osteoporosis is a progressive and irreversible disease when pathogeny persist, extending the treatment time of $\mathrm{Q}$ and its derivatives is merely conducive to delay the progression of osteoporosis rather than reverse it.

4.3.3. Possible Mechanisms. Systemic review of preclinical studies is conducive to understand comprehensively pathological mechanisms of disease and pharmacological effects of drugs [63]. We have summarized the possible mechanisms of $\mathrm{Q}$ and its derivatives mediated bone protection from cur- rent findings and listed them as follows: (1) Q alleviated oxidative damage by decreasing $\mathrm{NO}$ [36] and increasing GSH $[31,40]$, SOD $[39,45]$, and CAT [45] to reduce the release of MDA $[31,39]$ in the ovaries and bone tissue. A decreased MDA level in the ovaries directly increased estrogen activity [31] which has been shown to have antioxidant properties $[64,65]$. In addition, Gsk-3 $\beta / \mathrm{Nrf} 2$ signal pathway was reported to participate in the regulation of the abovementioned antioxidant process [39]. (2) Q and quercetin-3-Orutinose have the similar effect of phytoestrogen on inhibiting bone resorption by participating in the binding of estrogen receptor (ER) $[33,45,66]$, especially in $\operatorname{ER} \beta$ mainly expressed in bone [67]. However, another study [43] showed that $\mathrm{Q}$ did not appear to have this activity through either ER $\alpha$ or $\operatorname{ER} \beta$, which suggested that $\mathrm{Q}$ might affect bone metabolism through ERs independent pathway. (3) Q alleviated inflammatory reaction by inhibiting the expression of TNF$\alpha[4,32,36], \mathrm{IL}-6[4,36], \mathrm{INF}-\gamma[4]$, and CRP [36]. And then TNF- $\alpha$ activated NF- $\kappa \mathrm{B}$, increased the expression of NF- $\kappa \mathrm{B}$ protein, and promoted the degradation of $\beta$-catenin protein [31]. (4) $Q$ promoted bone synthesis by enhancing the expression of osteogenic protein (FoxO1, Bglap2, Collal, Osterix, and Runx2) [30, 39] via PI3K/Akt/Fox O1/NF- $\kappa \mathrm{B}$ signal pathway [30] and BMP2/smad4 signaling pathway [35]. (5) QCG inhibited the expression of osteoclast markers including RANK and c-fos in bone marrow cells (BMCs) cultured in the presence of RANK ligand and M-CSF [27]. $\mathrm{Q}$ and quercetin-3-O-beta-D-glucuronide inhibited RANKinduced osteoclast formation in a dose-dependent manner in RAW264.7 cells, and the RANK ligand-stimulated expression of osteoclast related genes including NFATc1 was inhibited by $\mathrm{Q}$ [43]. In addition, as the most abundant p38 
member in the bone and bone marrow [68], the ablation of p38 MAPK signaling in osteoblast lineage cells protects the mice from bone loss induced by estrogen deficiency [34, 69] found that Q could attenuate osteoporosis by downregulating MAPK signaling pathways. (6) Prostaglandins (PGs) played a role in IL-1-induced bone resorption [70]. Q was observed to reduce the production of PGs by inhibiting cyclooxygenase and phosphoesterase A2 [71]. The mechanism diagram is summarized in Figure 11.

\section{Conclusion}

The present study provided the preliminary preclinical evidence that oral administration of $\mathrm{Q}$ and its derivatives was capable of partially reversing osteopenia in animal models probably via antioxidant, anti-inflammatory, promoting osteogenesis, inhibiting osteoclasts, and its estrogen-like effect. The findings reveal the possibility of developing $Q$ and its derivatives as a drug or an ingredient in diet for the clinical treatment of osteoporosis.

\section{Conflicts of Interest}

The authors declare that there is no conflict of interests regarding the publication of this study.

\section{Authors' Contributions}

Yue-Yue Huang, Hong Wang, and Qun Zheng designed the study; Yue-Yue Huang, Hong Wang, and Qun Zheng collected the data; Yue-Yue Huang, Zi-Hao Wang, and Li-Hui Deng performed all analyses; All authors contributed to the writing of this manuscript. All the listed authors have read and approved the submitted manuscript. Yue-Yue Huang, $\mathrm{Zi}-\mathrm{Hao}$ Wang, and Li-Hui Deng contributed equally to this work.

\section{Acknowledgments}

This project was supported by the Clinical Research Foundation of the 2nd Affiliated Hospital of Wenzhou Medical University (SAHoWMU-CR2018-01-105), Lin He's New Medicine and Clinical Translation Academician Workstation Research Fund (17331208), Wenzhou Science and Technology Bureau Programs (H2015006 and Y20170322), and the Programs of Administration of Traditional Chinese Medicine in Zhejiang (2015ZB077 and 2018ZB080).

\section{References}

[1] J. E. Compston, M. R. McClung, and W. D. Leslie, "Osteoporosis," The Lancet, vol. 393, no. 10169, pp. 364-376, 2019.

[2] N. E. Lane, "Epidemiology, etiology, and diagnosis of osteoporosis," American Journal of Obstetrics and Gynecology, vol. 194, no. 2, pp. S3-S11, 2006.

[3] C. Cooper, G. Campion, and L. J. Melton III, "Hip fractures in the elderly: a world-wide projection," Osteoporosis International, vol. 2, no. 6, pp. 285-289, 1992.

[4] Z. Geng, L. L. Xu, X. Y. Zhang, and Y. G. Wang, "Effects of rutin on the prevention and treatment of osteoporosis in ovari- ectomized rats," Chinese Journal of Osteoporosis, vol. 25, pp. 497-501, 2019.

[5] R. K. Evans, C. H. Negus, A. J. Centi, B. A. Spiering, W. J. Kraemer, and B. C. Nindl, "Peripheral QCT sector analysis reveals early exercise induced increases in tibial bone mineral density," Journal of Musculoskeletal \& Neuronal Interactions, vol. 12, no. 3, pp. 155-164, 2012.

[6] D. C. Bauer, "Calcium supplements and fracture prevention," The New England Journal of Medicine, vol. 369, no. 16, pp. 1537-1543, 2013.

[7] S. L. Kopecky, D. C. Bauer, M. Gulati et al., "Lack of evidence linking calcium with or without vitamin D supplementation to cardiovascular disease in generally healthy adults: a clinical guideline from the National Osteoporosis Foundation and the American Society for Preventive Cardiology," Annals of Internal Medicine, vol. 165, no. 12, pp. 867-868, 2016.

[8] M. H. Murad, M. T. Drake, R. J. Mullan et al., "Comparative effectiveness of drug treatments to prevent fragility fractures: a systematic review and network meta-analysis," The Journal of Clinical Endocrinology and Metabolism, vol. 97, no. 6, pp. 1871-1880, 2012.

[9] J. A. Cauley, J. Robbins, Z. Chen et al., "Effects of estrogen plus progestin on risk of fracture and bone mineral density: the Women's Health Initiative randomized trial," Journal of the American Medical Association, vol. 290, no. 13, pp. 17291738, 2003.

[10] C. J. Crandall, S. J. Newberry, A. Diamant et al., "Comparative effectiveness of pharmacologic treatments to prevent fractures: an updated systematic review," Annals of Internal Medicine, vol. 161, no. 10, pp. 711-723, 2014.

[11] S. R. Cummings, J. San Martin, M. R. McClung et al., "Denosumab for prevention of fractures in postmenopausal women with osteoporosis," The New England Journal of Medicine, vol. 361, no. 8, pp. 756-765, 2009.

[12] R. M. Neer, C. D. Arnaud, J. R. Zanchetta et al., "Effect of parathyroid hormone (1-34) on fractures and bone mineral density in postmenopausal women with osteoporosis," The New England Journal of Medicine, vol. 344, no. 19, pp. 1434-1441, 2001.

[13] D. Tang, C. Ju, Y. Liu, F. Xu, Z. Wang, and D. Wang, “Therapeutic effect of icariin combined with stem cells on postmenopausal osteoporosis in rats," Journal of Bone and Mineral Metabolism, vol. 36, no. 2, pp. 180-188, 2018.

[14] A. P. Hermann and B. Abrahamsen, "The bisphosphonates: risks and benefits of long term use," Current Opinion in Pharmacology, vol. 13, no. 3, pp. 435-439, 2013.

[15] J. R. Lewis, M. Sim, and R. M. Daly, "The vitamin D and calcium controversy: an update," Current Opinion in Rheumatology, vol. 31, no. 2, pp. 91-97, 2019.

[16] M. J. Bolland, A. Avenell, J. A. Baron et al., "Effect of calcium supplements on risk of myocardial infarction and cardiovascular events: meta-analysis," BMJ, vol. 341, article c6923, 2010.

[17] M. J. Bolland, A. Grey, A. Avenell, G. D. Gamble, and I. R. Reid, "Calcium supplements with or without vitamin D and risk of cardiovascular events: reanalysis of the Women's Health Initiative limited access dataset and meta-analysis," BMJ, vol. 342, article d2040, 2011.

[18] B. Dawson-Hughes and S. S. Harris, "High-dose vitamin D supplementation: too much of a good thing?," Journal of the American Medical Association, vol. 303, no. 18, pp. 18611862, 2010. 
[19] K. E. Hansen, "High-dose vitamin D: helpful or harmful?," Current Rheumatology Reports, vol. 13, no. 3, pp. 257-264, 2011.

[20] H. Gronier and G. Robin, "Cardiovascular risks of combined oral contraceptives - beyond the French controversy," Gynécologie, Obstétrique \& Fertilité, vol. 42, pp. 174-181, 2014.

[21] K. L. Dennison, N. B. Samanas, Q. E. Harenda et al., "Development and characterization of a novel rat model of estrogeninduced mammary cancer," Endocrine-Related Cancer, vol. 22, no. 2, pp. 239-248, 2015.

[22] Q. Gan, S. Crumley, and R. R. Broaddus, "Molecular modifiers of hormone receptor action: decreased androgen receptor expression in mismatch repair deficient endometrial endometrioid adenocarcinoma," International Journal of Gynecological Pathology, vol. 38, no. 1, pp. 44-51, 2019.

[23] M. Koszykowska, J. Calka, P. Szwajca, and B. Jana, "Long-term estradiol-17 $\beta$ administration decreases the number of neurons in the caudal mesenteric ganglion innervating the ovary in sexually mature gilts," The Journal of Reproduction and Development, vol. 57, no. 1, pp. 62-71, 2011.

[24] N. Oršolić, E. Goluža, D. Đikić et al., "Role of flavonoids on oxidative stress and mineral contents in the retinoic acidinduced bone loss model of rat," European Journal of Nutrition, vol. 53, no. 5, pp. 1217-1227, 2014.

[25] Y. X. Yu, S. R. Ge, and G. Z. Wang, "Research progress in the pharmacological action of quercetin and its derivatives," Journal of Chinese Medicinal Materials, vol. 26, pp. 902-904, 2003.

[26] R. W. K. Wong and A. B. M. Rabie, "Effect of quercetin on preosteoblasts and bone defects," The Open Orthopaedics Journal, vol. 2, no. 1, pp. 27-32, 2008.

[27] J. A. Siddiqui, K. Sharan, G. Swarnkar et al., "Quercetin-6-C- $\beta$ D-glucopyranoside isolated from Ulmus wallichiana planchon is more potent than quercetin in inhibiting osteoclastogenesis and mitigating ovariectomy-induced bone loss in rats," Menopause, vol. 18, no. 2, pp. 1-207, 2011.

[28] J. van Luijk, M. Leenaars, C. R. Hooijmans, K. E. Wever, R. B. de Vries, and M. Ritskes-Hoitinga, "Towards evidence-based translational research: the pros and cons of conducting systematic reviews of animal studies," ALTEX, vol. 30, no. 2, pp. 256-257, 2013.

[29] M. R. Macleod, T. O’Collins, D. W. Howells, and G. A. Donnan, "Pooling of animal experimental data reveals influence of study design and publication bias," Stroke, vol. 35, no. 5, pp. 1203-1208, 2004.

[30] J. Min, Effect of Quercetin on Bone Metabolism in Ovarian Ovariectomized Rats and its Mechanism, [Ph.D thesis], Nanchang University, 2019.

[31] N. Oršolić, Ž. Jeleč, J. Nemrava, V. Balta, G. Gregorović, and D. Jeleč, "Effect of quercetin on bone mineral status and markers of bone turnover in retinoic acid-induced osteoporosis," Polish Journal of Food and Nutrition Sciences, vol. 68, no. 2, pp. 149-162, 2018.

[32] Z. Yuan, J. Min, Y. Zhao et al., "Quercetin rescued TNF-alphainduced impairments in bone marrow-derived mesenchymal stem cell osteogenesis and improved osteoporosis in rats," American Journal of Translational Research, vol. 10, pp. 4313-4321, 2018.

[33] Z. Yuan, J. Min, K. Wang, F. Long, Y. J. Wang, and J. Luo, "Effects of different pharmacological components of eucommia ulmoides flavonoids on postmenopausal osteoporosis," Chinese Journal of Osteoporosis, vol. 24, 2018.
[34] L. Z. Xing, H. J. Ni, and Y. L. Wang, "Quercitrin attenuates osteoporosis in ovariectomized rats by regulating mitogenactivated protein kinase (MAPK) signaling pathways," Biomedicine \& Pharmacotherapy, vol. 89, pp. 1136-1141, 2017.

[35] H. Zheng, W. Tang, J. L. Jiao, w. Chao, X. Yuan et al., "Molecular mechanism of quercetin in the treatment of ovariectomized osteoporosis rats by promoting osteogenic differentiation," Pharmacology and Clinics of Chinese Materia Medica, vol. 33, pp. 16-20, 2017.

[36] H. M. Abdelkarem, L. M. Fadda, and O. R. Kaml, "Alleviation of bone markers in rats induced nano-zinc oxide by qurecetin and $\alpha$-lipolic acid," Toxicology Mechanisms and Methods, vol. 26, no. 9, pp. 692-699, 2016.

[37] W. Bian, H. Sun, K. Liu, L. Yang, and R. H. Zhang, "Effects of quercetin on the aspcts of bone biomechanical properties and metabolism in osteoporotic rats," Jilin Journal of Traditional Chinese Medicine, vol. 36, 2016.

[38] J. J. Feng, H. Zou, H. Y. Zhu, and Y. Gan, "Effect of quercetin on serum osteocalcin levels and femur type I collagen protein levels in the ovariectomized rats," Journal of Traditional Chinese Orthopedics and Traumatology, vol. 28, 2016.

[39] Y. Z. Zhou, Effects of high-fat diet and quercetin intervention on bone performance of obesity prone and obesity resistant mice, [Ph.D thesis], Jiangnan University, 2016, https://kns .cnki.net $/ \mathrm{KCMS} /$ detail/detail.aspx ?dbcode $=\mathrm{CMFD} \& \mathrm{db}$ name $=$ CMFD2.

[40] Y. J. Tian, L. N. Li, J. Sun, J. Zhen, G. W. Le, and Y. H. Shi, "Effects of resveratrol and quercetin on femur performance in mice fed high-fat diet," Acta Nutrimenta Sinica, vol. 36, pp. 366-370, 2014.

[41] H. Derakhshanian, M. Djalali, A. Djazayery et al., "Quercetin prevents experimental glucocorticoid-induced osteoporosis: a comparative study with alendronate," Canadian Journal of Physiology and Pharmacology, vol. 91, no. 5, pp. 380-385, 2013.

[42] W. Liang, Z. Luo, S. Ge et al., "Oral administration of quercetin inhibits bone loss in rat model of diabetic osteopenia," European Journal of Pharmacology, vol. 670, no. 1, pp. 317-324, 2011.

[43] M. Tsuji, H. Yamamoto, T. Sato et al., "Dietary quercetin inhibits bone loss without effect on the uterus in ovariectomized mice," Journal of Bone and Mineral Metabolism, vol. 27, no. 6, pp. 673-681, 2009.

[44] Y. Wang, "Effects of quercetin on reproductive system and bone mineral density in ovariectomized rats," China Practical Medicine, vol. 3.

[45] X. J. Zhu and S. Q. Wei, "Protective effect of quercetin on ovariectomy-induced bone loss in rats," Chinese Journal of Osteoporosis, vol. 11, pp. 504-508, 2008.

[46] M. N. Horcajada-Molteni, V. Crespy, V. Coxam, M. J. Davicco, C. Rémésy, and J. P. Barlet, "Rutin inhibits ovariectomyinduced osteopenia in rats," Journal of Bone and Mineral Research, vol. 15, no. 11, pp. 2251-2258, 2000.

[47] D. Moher, M. Avey, G. Antes, and D. G. Altman, "Erratum: the national institutes of health and guidance for reporting preclinical research," BMC Medicine, vol. 13, no. 1, pp. 17417015, 2015.

[48] M. Permuy, M. López-Peña, F. Muñoz, and A. GonzálezCantalapiedra, "Rabbit as model for osteoporosis research," Journal of Bone and Mineral Metabolism, vol. 37, no. 4, pp. 573-583, 2019. 
[49] S. T. Rashid, H. J. Salacinski, G. Hamilton, and A. M. Seifalian, "The use of animal models in developing the discipline of cardiovascular tissue engineering: a review," Biomaterials, vol. 25, no. 9, pp. 1627-1637, 2004.

[50] E. Martin-Monge, I. F. Tresguerres, L. Blanco, A. Khraisat, R. Rodríguez-Torres, and J. A. Tresguerres, "Validation of an osteoporotic animal model for dental implant analyses: an in vivo densitometric study in rabbits," The International Journal of Oral \& Maxillofacial Implants, vol. 26, no. 4, pp. 725730, 2011.

[51] B. Schröder, S. Vössing, and G. Breves, "In vitro studies on active calcium absorption from ovine rumen," Journal of Comparative Physiology B: Biochemical, Systemic, and Environmental Physiology, vol. 169, no. 7, pp. 487-494, 1999.

[52] S. Reinwald and D. Burr, "Review of nonprimate, large animal models for osteoporosis research," Journal of Bone and Mineral Research, vol. 23, no. 9, pp. 1353-1368, 2008.

[53] R. Brommage, "Perspectives on using nonhuman primates to understand the etiology and treatment of postmenopausal osteoporosis," Journal of Musculoskeletal \& Neuronal Interactions, vol. 1, no. 4, pp. 307-325, 2001.

[54] M. Egermann, J. Goldhahn, and E. Schneider, "Animal models for fracture treatment in osteoporosis," Osteoporosis International, vol. 16, no. S02, pp. S129-S138, 2005.

[55] R. T. Turner, A. Maran, S. Lotinun et al., “Animal models for osteoporosis," Reviews in Endocrine \& Metabolic Disorders, vol. 2, no. 1, pp. 117-127, 2001.

[56] S. Castañeda, E. Calvo, R. Largo et al., "Characterization of a new experimental model of osteoporosis in rabbits," Journal of Bone and Mineral Metabolism, vol. 26, no. 1, pp. 53-59, 2008.

[57] M. R. Zarrinkalam, H. Beard, C. G. Schultz, and R. J. Moore, "Validation of the sheep as a large animal model for the study of vertebral osteoporosis," European Spine Journal, vol. 18, no. 2, pp. 244-253, 2009.

[58] R. Oheim, F. T. Beil, T. Köhne et al., "Sheep model for osteoporosis: sustainability and biomechanical relevance of low turnover osteoporosis induced by hypothalamic-pituitary disconnection," Journal of Orthopaedic Research, vol. 31, no. 7, pp. 1067-1074, 2013.

[59] D. Arens, I. Sigrist, M. Alini, P. Schawalder, E. Schneider, and M. Egermann, "Seasonal changes in bone metabolism in sheep," The Veterinary Journal, vol. 174, no. 3, pp. 585-591, 2007.

[60] A. D. Cardon, M. R. Bailey, and B. T. Bennett, "The Animal Welfare Act: from enactment to enforcement," Journal of the American Association for Laboratory Animal Science, vol. 51, pp. 301-305, 2012.

[61] Qi, “Advances in animal models of osteoporosis," Heilongjiang Journal of Animal Science and Veterinary Medicine, vol. 9, pp. 96-98, 2016.

[62] C. Manach, G. Williamson, C. Morand, A. Scalbert, and C. Rémésy, "Bioavailability and bioefficacy of polyphenols in humans. I. Review of 97 bioavailability studies," The American Journal of Clinical Nutrition, vol. 81, no. 1, pp. 230S-242S, 2005.

[63] D. G. Hackam and D. A. Redelmeier, "Translation of research evidence from animals to humans," Journal of the American Medical Association, vol. 296, no. 14, pp. 1731-1732, 2006.

[64] F. Sohair and M. S. Amel, "Oxidative stress as a risk factor of osteoporotic model induced by vitamin A in rats. 2009," Aus- tralian Journal of Basic and Applied Sciences, vol. 3, pp. 15591568, 2009.

[65] C. Prouillet, J. C. Mazière, C. Mazière, A. Wattel, M. Brazier, and S. Kamel, "Stimulatory effect of naturally occurring flavonols quercetin and kaempferol on alkaline phosphatase activity in MG-63 human osteoblasts through ERK and estrogen receptor pathway," Biochemical Pharmacology, vol. 67, no. 7, pp. 1307-1313, 2004.

[66] C. Castelo-Branco and I. Soveral, "Phytoestrogens and bone health at different reproductive stages," Gynecological Endocrinology, vol. 29, no. 8, pp. 735-743, 2013.

[67] D. Seidlová-Wuttke, K. Prelle, K. H. Fritzemeier, and W. Wuttke, "Effects of estrogen receptor alpha- and betaselective substances in the metaphysis of the tibia and on serum parameters of bone and fat tissue metabolism of ovariectomized rats," Bone, vol. 43, no. 5, pp. 849-855, 2008.

[68] M. B. Greenblatt, J. H. Shim, W. Zou et al., "The p38 MAPK pathway is essential for skeletogenesis and bone homeostasis in mice," The Journal of Clinical Investigation, vol. 120, no. 7, pp. 2457-2473, 2010.

[69] C. Thouverey and J. Caverzasio, "Ablation of p38 $\alpha$ MAPK signaling in osteoblast lineage cells protects mice from bone loss induced by estrogen deficiency," Endocrinology, vol. 156, no. 12, pp. 4377-4387, 2015.

[70] Y. Onoe, C. Miyaura, and T. Kaminakayashiki, "IL-13 and IL14 inhibit bone resorption by suppressing cyclooxygenase-2dependent prostaglandin synthesis in osteoblasts," The Journal of Immunology, vol. 156, pp. 758-764, 1996.

[71] G. J. Soleas, E. P. Diamandis, and D. M. Goldberg, "Wine as a biological fluid: history, production, and role in disease prevention," Journal of Clinical Laboratory Analysis, vol. 11, no. 5, pp. 287-313, 1997. 